\title{
Acyloxylation of Cyclic Enones: Synthesis of Densely Oxygenated Guaianolides
}

\author{
Rubén Marín-Barrios, Ana Leticia García-Cabeza, F. Javier Moreno-Dorado,* Francisco M. Guerra,* \\ and Guillermo M. Massanet
}

Departamento de Química Orgánica, Facultad de Ciencias, Universidad de Cádiz, 11510 Puerto Real, Cádiz, Spain

Supporting Information

ABSTRACT: The $\alpha^{\prime}$-acyloxylation of cyclic enones with linear carboxylic acids is described. The reaction is promoted by $\mathrm{KMnO}_{4}$ in the presence of a carboxylic acid and its corresponding carboxylic anhydride. The optimization of the reaction has been carried out using the statistical methodology known as design of experiments. The optimized reaction conditions have been evaluated in terms of substrate scope and

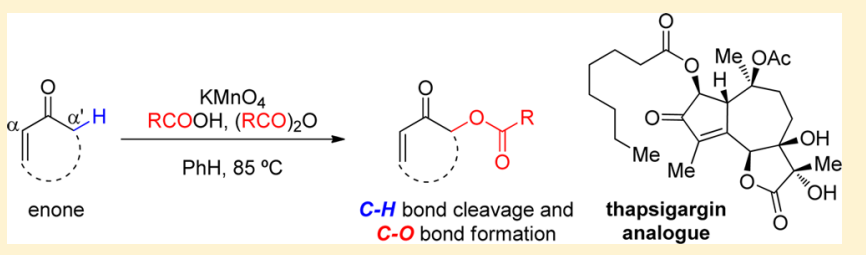
compatibility with different functional groups. The methodology has been applied to the synthesis of densely oxygenated guaianes and guaianolides.

\section{INTRODUCTION}

In the last decades, different methodologies for the activation of $\mathrm{C}-\mathrm{H}$ bonds have emerged. ${ }^{1}$ Remarkable progress has been made in the $\mathrm{C}\left(\mathrm{sp}^{2}\right)-\mathrm{H}$ activation (aromatic or vinylic) ${ }^{2}$ and in the oxidation of $\mathrm{C}\left(\mathrm{sp}^{3}\right)-\mathrm{H}$ bonds at allylic positions. ${ }^{3}$ Another interesting reaction involving the transformation of $\mathrm{C}\left(\mathrm{sp}^{3}\right)-\mathrm{H}$ bonds is the $\alpha$-oxidation of a carbonyl group. This kind of reaction has been the subject of research since many biologically significant compounds bear either hydroxyl or acyloxy groups in this position. ${ }^{4}$

In 1989, Watt et al. reported that enones could be functionalized at the $\alpha^{\prime}$-position by treatment with $\mathrm{Mn}(\mathrm{OAc})_{3}$ in the presence of different carboxylic acids. ${ }^{5}$ The mechanism involves the in situ generation of a radical Mn(III) carboxylate, which reacts with the enone, leading to the corresponding $\alpha^{\prime}$ acyloxyenone. ${ }^{6}$ Although good yields were reported, the methodology presented some problems: (i) the need for a large excess of the carboxylic acid and the Mn precursor, which, in addition, had to be freshly prepared ${ }^{7}$ or dried prior to use; (ii) the use of a Dean-Stark trap to remove the water, which made scaling up or down the reaction difficult; and (iii) poor functional group compatibility.

Demir et al. reported some improvements by using $\mathrm{KMnO}_{4}$ instead of $\mathrm{Mn}(\mathrm{OAc})_{3}$ to generate the $\mathrm{Mn}(\mathrm{III})$ species in situ. ${ }^{8}$ Yet, the scope of the reaction was rather limited and the application of this methodology to the synthesis of natural products remained a challenge. References applying this method into complex syntheses are lacking, and the reproducibility issues and some contradictory results have prevented this reaction to be included in the pool of synthetically useful reactions.

Herein, we present our study and optimization of the $\alpha^{\prime}$ acyloxylation of cyclic enones by $\mathrm{KMnO}_{4}$ in the presence of a mixture of a carboxylic acid and its corresponding anhydride as an effective water scavenger. The use of Dean-Stark apparatus is no longer necessary, simplifying the scaling of the reaction. The reaction conditions have been optimized using a design of experiments (DoE) approach, ${ }^{9}$ which guarantees the robustness of the method. The optimized reaction conditions have been applied to the synthesis of several guaianes and guaianolides, closely related to thapsigargin $\mathbf{1 a}$ and thapsigargicin $\mathbf{1 b}$, two compounds with remarkable anti-SERCA ATPase activity (Figure 1). ${ }^{10}$

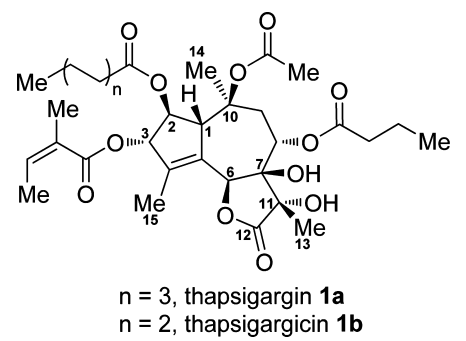

Figure 1. Thapsigargin 1a and thapsigargicin $\mathbf{1 b}$.

\section{RESULTS AND DISCUSSION}

In the seminal report by Watt, it was demonstrated that removal of water was necessary for the reaction to proceed. ${ }^{5}$ When we first ran the reaction using small amounts of the reactants, the Dean-Stark trap was not effective. The collected solvent in the side arm of the trap was cloudy, but the amount of water generated was too small to become an isolated aqueous layer. To solve this problem and simplify the reaction setup, we undertook an exploratory screening of methods to remove efficiently the generated water. As a benchmark

Received: April 25, 2014

Published: June 17, 2014 
Table 1. Screening of Conditions without a Dean-Stark Apparatus ${ }^{a}$

\begin{tabular}{|c|c|c|c|c|c|c|}
\hline entry & time prior to substrate addition ( $\min )$ & temp $\left({ }^{\circ} \mathrm{C}\right)$ & $\mathrm{KMnO}_{4}$ (equiv) & $\mathrm{AcOH}$ (equiv) & additive & yield $^{b}(\%)$ \\
\hline 1 & 60 & 85 & 1.20 & 10 & $4 \AA \mathrm{MS}(0.060 \mathrm{~g})$ & 39 \\
\hline 2 & 30 & 85 & 3.10 & 25 & $4 \AA ̊ \mathrm{MS}(0.230 \mathrm{~g})$ & 10 \\
\hline 3 & 60 & 50 & 2.10 & 12 & $4 \AA$ MS $(0.230 \mathrm{~g})$ & 1 \\
\hline 4 & 45 & 65 & 2.10 & 17 & $4 \AA$ MS $(0.140 \mathrm{~g})$ & 12 \\
\hline 5 & 60 & 85 & 3.10 & 25 & $4 \AA \mathrm{MS}(0.230 \mathrm{~g})$ & 30 \\
\hline 6 & 45 & 65 & 2.10 & 170 & $4 \AA ̊ \mathrm{MS}(0.140 \mathrm{~g})$ & 17 \\
\hline 7 & 30 & 85 & 3.10 & 170 & $\mathrm{Na}_{2} \mathrm{SO}_{4}$ (10 equiv) & 37 \\
\hline 8 & 30 & 85 & 3.10 & 170 & $\mathrm{Ac}_{2} \mathrm{O}$ (100 equiv) & 63 \\
\hline 9 & 30 & 85 & 3.10 & 170 & $\mathrm{AcCl}$ (100 equiv) & 32 \\
\hline
\end{tabular}

${ }^{a}$ Reaction conditions: $\mathrm{KMnO}_{4}, \mathrm{AcOH}$, additive, and $\mathrm{PhH}(4 \mathrm{~mL})$ at the stated temperature and stir for the indicated time, then isophorone 3 (0.025 $\mathrm{mL}, 0.160 \mathrm{mmol}$ ) and stir overnight at the stated temperature. ${ }^{b}$ Determined by GC analysis.

reaction, we chose the $\alpha^{\prime}$-acetoxylation of isophorone 3 to produce 6-acetylisophorone 4 . We considered five variables to study: (i) time prior to the substrate addition, needed for the formation of the radical $\mathrm{Mn}$ (III) carboxylate, (ii) temperature, (iii) equivalents of $\mathrm{KMnO}_{4}$, (iv) equivalents of acetic acid, and (v) amount of $4 \AA$ molecular sieves. As illustrated in Table 1 (entries 1-6), the use of $4 \AA$ molecular sieves resulted in poor yields presumably due to the high temperature. ${ }^{11}$ Other additives were also evaluated for the same role, among them acetic anhydride. The reduction of $\mathrm{Mn}(\mathrm{VII})$ to $\mathrm{Mn}$ (III) involves the formation of water. ${ }^{8}$ The use of a carboxylic anhydride might favor the reaction by removing water and serve as well as an acyloxy group source, along with the carboxylic acid itself. As expected, higher yields were obtained when acetic anhydride was used in conjunction with acetic acid (entry 8, Table 1).

Since the actual mechanism of the reaction remained unclear, we considered the use of a design of experiments (DoE) approach for the optimization of the reaction to be convenient. DoE is a statistical optimization method that explores an experimental domain, setting up different variables or factors in high, medium, and low levels. The influence of these variables is thus studied, and the response (yield in our case) is optimized. Instead of optimizing one variable at a time (an OVAT approach), DoE performs the optimization of several variables simultaneously. An algorithm suggests the conditions in which the experiments have to be run. The response is evaluated, and a model for the behavior of the system is obtained. ${ }^{9}$ The aim is to get the maximum amount of information using the minimum number of experiments.

Our model would take into account three variables: (i) equivalents of $\mathrm{KMnO}_{4}$, (ii) equivalents of acetic acid, and (iii) equivalents of acetic anhydride. To ensure the effective formation of the radical $\mathrm{Mn}$ (III) carboxylate, the time prior to the addition of the substrate and the temperature were set at $30 \mathrm{~min}$ and $85{ }^{\circ} \mathrm{C}$, respectively. Finally, the substrate concentration was kept at $0.04 \mathrm{M}$ in benzene. We decided to perform a Box-Behnken design (BBD), with three levels for each variable (Table 2). The experiments were carried out according to the matrix shown in Table 3. The yields were measured, and a quadratic model was fitted for the reaction. The variance of the method was estimated by running three replicates of the central point (Table 3, entries 4, 8, 10, and 11).
Table 2. Selected Levels for Each Variable for BBD

\begin{tabular}{lcccc} 
& & \multicolumn{3}{c}{ levels } \\
\cline { 3 - 5 } variables & symbol & low & medium & high \\
equiv of $\mathrm{KMnO}_{4}$ & $\mathrm{~A}$ & 1.05 & 2.10 & 3.15 \\
equiv of $\mathrm{AcOH}$ & $\mathrm{B}$ & 10.0 & 22.5 & 35.0 \\
equiv of $\mathrm{Ac}_{2} \mathrm{O}$ & $\mathrm{C}$ & 5.00 & 9.50 & 14.0 \\
\hline
\end{tabular}

Table 3. Design of Experiments Optimization for $\boldsymbol{\alpha}^{\prime}$ Acetyloxylation of Isophorone $3^{a, b}$

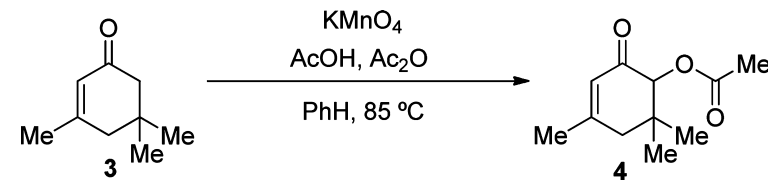

\begin{tabular}{rcccc} 
& \multicolumn{4}{c}{ variables } \\
\cline { 2 - 4 } run & $\mathrm{A}$ & $\mathrm{B}$ & $\mathrm{C}$ & \\
\cline { 2 - 4 } 1 & 3.15 & 22.5 & 5.00 & 45 \\
2 & 3.15 & 10.0 & 9.50 & 34 \\
3 & 1.05 & 35.0 & 9.50 & 86 \\
4 & 2.10 & 22.5 & 9.50 & 56 \\
5 & 1.05 & 22.5 & 5.00 & 63 \\
6 & 3.15 & 22.5 & 14.0 & 51 \\
7 & 2.10 & 35.0 & 5.00 & 71 \\
8 & 2.10 & 22.5 & 9.50 & 69 \\
9 & 3.15 & 35.0 & 9.50 & 67 \\
10 & 2.10 & 22.5 & 9.50 & 75 \\
11 & 2.10 & 22.5 & 9.50 & 65 \\
12 & 2.10 & 10.0 & 14.0 & 48 \\
13 & 2.10 & 10.0 & 5.00 & 40 \\
14 & 2.10 & 35.0 & 9.50 & 91 \\
15 & 1.05 & 22.5 & 14.0 & 58 \\
16 & 1.05 & 10.0 & 9.50 & 62 \\
17 & 2.10 & 35.0 & 14.0 & 73
\end{tabular}

${ }^{a}$ Reaction conditions: $\mathrm{KMnO}_{4}$ (variable A), $\mathrm{AcOH}(\mathrm{B})$, and $\mathrm{Ac}_{2} \mathrm{O}(\mathrm{C})$ in the indicated amounts, and $\mathrm{PhH}(4 \mathrm{~mL})$ at $85{ }^{\circ} \mathrm{C}$ for $30 \mathrm{~min}$, then isophorone $3(0.025 \mathrm{~mL}, 0.160 \mathrm{mmol})$, stirring at $85{ }^{\circ} \mathrm{C}$ overnight. ${ }^{b}$ Runs displayed in the order determined by the Box-Behnken matrix. ${ }^{c}$ Determined by GC analysis.

According to the results, the optimal conditions were those corresponding to 2.10 equiv of $\mathrm{KMnO}_{4}, 35.0$ equiv of acetic 
acid, and 9.50 equiv of acetic anhydride, which afforded a $91 \%$ yield by GC analysis (entry 14, Table 3; $100 \%$ isolated yield). As an alternative to the use of benzene, other solvents were evaluated. Moderate yields were obtained with dichloroethane (62\%) and toluene (59\%). Cyclohexene and acetonitrile afforded lower yields (45\% and 35\%, respectively).

The obtained model is illustrated by the calculated response surface (Figure 2), which represents the dependence of the

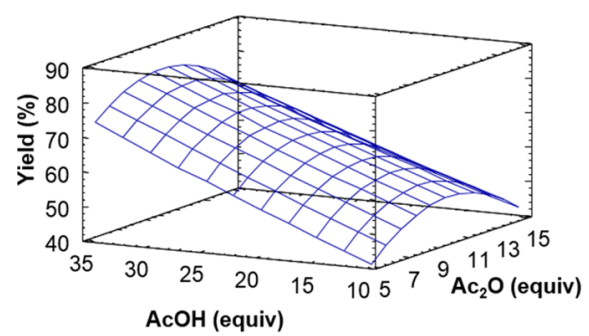

Figure 2. Calculated response surface for the yield of $\mathbf{4}$ (medium level of $\mathrm{KMnO}_{4}, 2.10$ equiv).

yield on the studied variables. An inspection of Figure 2 shows that, for an intermediate level of $\mathrm{KMnO}_{4}$, the combination of an intermediate level of acetic anhydride with high levels of acetic acid would lead to higher responses. In addition, the Pareto plot confirms the influence of the acetic anhydride in the system, since its quadratic effect (CC) is significant at $90 \%$ confidence (Figure 3). Other interaction effects among variables $(\mathrm{AA}, \mathrm{AC}, \mathrm{AB}, \mathrm{BC}$, and $\mathrm{BB})$ were not significant. As expected, higher amounts of acetic acid lead to higher yields.

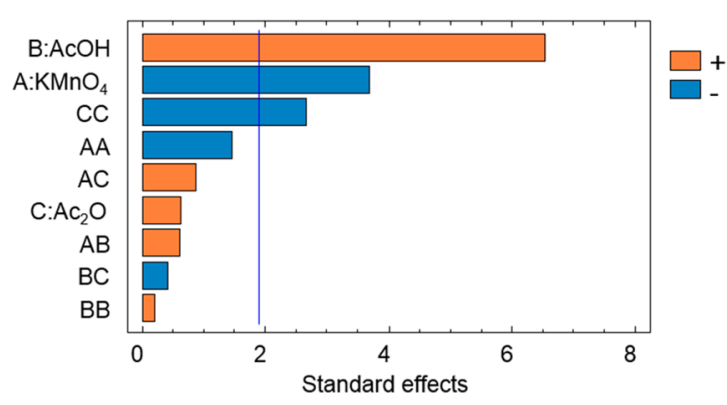

Figure 3. Pareto plot for standard effects over the yield of 4 .

The statistical significance of the model was evaluated by analysis of variance (ANOVA) and the calculation of the coefficient of determination $R^{2}$ (Table 4). Although the $F$ value of the model, 3.46, was not high enough to ensure significance at $90 \%$ confidence ( $F$ was lower than the critical $F$ value, 3.90), the $R^{2}$ coefficient was 0.9122 , which implied a good fit between predicted and experimental data. The model explained the $95.51 \%$ of the variance. Furthermore, the $F$ value for the lack of fit, $2.49\left(<F_{c}\right)$, indicated that it was not significant relative to pure error. These results allowed us to consider the model as robust and the obtained optimal conditions as reproducible.

The scope of the reaction was further investigated. Several substrates and linear carboxylic acids with chains of different lengths $(2,4,6$, and 8 carbon atoms) were selected (Table 5). The yields ranged from good to excellent, but no trend attributable to the substrate or the chain length was observed.

We next turned to study the reaction with more complex substrates. Guaianes constitute a widely distributed group of sesquiterpenes, many of which bear a cyclopentenone system. Most guaianolides isolated from Umbelliferae plants display an acyl group located at the C-2 carbon atom, which we envisaged that could be set by $\alpha^{\prime}$-oxidation of a ketone previously located at C-3. They seemed a suitable scaffold for the testing of the reaction in a more complex environment. We then decided to test guaianolide $\mathbf{2 1}$ and guaiane $\mathbf{2 5}$ as substrates for this methodology.

O-Acetylisophotosantonic lactone $\mathbf{2 1}$ was easily prepared by photochemical rearrangement in 38\% yield from $\alpha$-santonin $\mathbf{2 0}$ using $\mathrm{AcOH}$ as the solvent (Scheme 1a). ${ }^{12}$ On the other hand, guaiane $\mathbf{2 5}$ is an intermediate that can be prepared by following a methodology that we devised for the synthesis of analogues of thapsigargin. ${ }^{13}$ To this end, compound 23 was subjected to photochemical rearrangement in $\mathrm{AcOH}$, providing 24 in $72 \%$ yield. Subsequent protection of the primary hydroxyl group with TBDMSCl and imidazole afforded guaiane 25 in almost quantitative yield (Scheme 1b).

The behavior of compounds $\mathbf{2 1}$ and $\mathbf{2 5}$ under the oxidation conditions demonstrated the tolerance of the reaction to the presence of different functional groups such as lactones, esters, double bonds, and silyl protected hydroxyl groups (Table 6). The reaction outcome was affected by substrate stereochemical control, since one single diastereomer was detected in all cases. Structural features in derivatives 26-29 make them interesting from a biological perspective since many sesquiterpene lactones are lead compounds in drug discovery. ${ }^{14}$

Our interest in bioactive guaianolides prompted us to apply this methodology to the synthesis of new analogues of thapsigargin $\mathbf{1 a}$ and thapsigargicin $\mathbf{1 b}$. There is just one reported total synthesis of thapsigargin and several members of the family by Ley et al. ${ }^{15}$ Some unnatural derivatives have been also synthesized by the same authors. ${ }^{16}$ Currently, a prodrug ${ }^{17}$ against prostate cancer based on thapsigargin is being tested in clinical trials. ${ }^{18}$ The remarkable biological activities of thapsigargin and related compounds and the scarcity of methods for their preparation make the opening of new routes of synthesis interesting.

Compound 33 could be conveniently modified to provide thapsigargin analogues (Scheme 2). To this end, 33 was prepared from 25 on a $1 \mathrm{~g}$ scale with good yield (see the Experimental Section). Then, it was submitted to TBAF

Table 4. ANOVA Results of the Quadratic Model for the Yield of $4^{a}$

$\begin{array}{lccc}\text { source } & \text { sum of squares } & \text { degree of freedom } & \text { mean squares } \\ \text { total } & 3861.87 & 16 & F \text { values } \\ \text { model } & 3522.67 & 12 & 3.46 \\ \text { residual } & 339.20 & 4 & 84.80 \\ \text { lack of fit } & 153.86 & 1 & 153.86 \\ \text { pure error } & 185.35 & 3 & 61.78\end{array}$

${ }^{a} R^{2}=0.9122 ; R=0.9551 .95 .51 \%$ of the variance explained by the model. 
Table 5. Scope of the Reaction of $\alpha^{\prime}$-Acyloxylation of Enones ${ }^{a, b}$
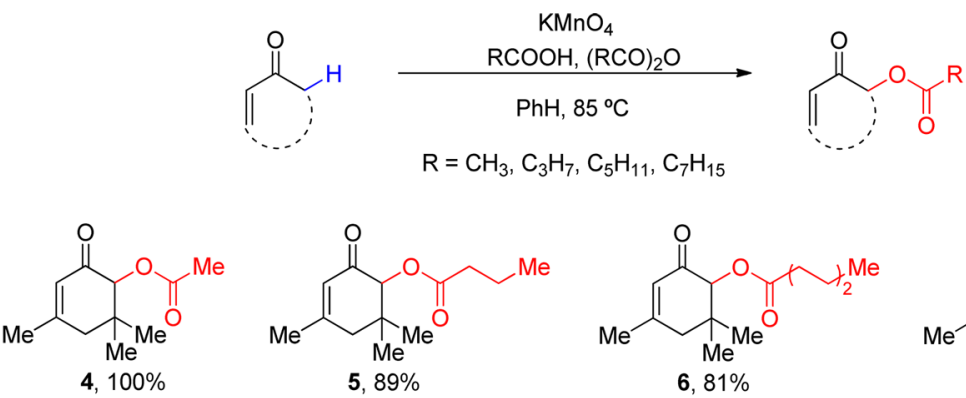<smiles>CCCC(=O)OC1C(=O)C=C(C)CC1(C)C</smiles><smiles>CCCC(=O)OC1C(=O)C=C(C)CC1(C)C</smiles><smiles>CCCC(=O)OC1C(=O)C=C(C)CC1(C)C</smiles><smiles>CC(=O)OC1CCC([18O])CC1=O</smiles>

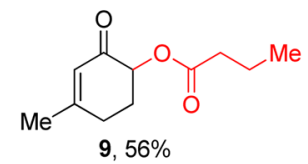<smiles>CCCC(=O)OC1CCC(C)=CC1=O</smiles>

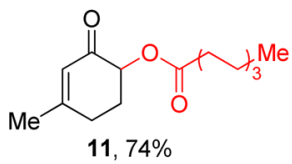<smiles>CCCCCC1=C(C)CC(OC(C)=O)C1=O</smiles><smiles>CCCCC1=C(C)CC(OC(=O)CCC)C1=O</smiles><smiles>CCCCC1=C(C)CC(OC(=O)CCC)C1=O</smiles>

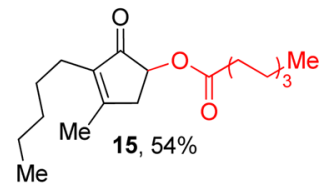<smiles>CCOC1=CC(=O)C(OC(C)=O)CC1</smiles><smiles>CCOC1=CC(=O)C(OC(=O)CC[18F])CC1</smiles><smiles>CCCC(=O)OC1CCC(OCC)=CC1=O</smiles><smiles>CCCCCCCCCCCC(=O)OC1CCC(OCC)=CC1=O</smiles>

${ }^{a}$ Reaction conditions: $\mathrm{KMnO}_{4}(83 \mathrm{mg}, 0.525 \mathrm{mmol}, 2.10$ equiv), carboxylic acid (35.0 equiv), and carboxylic anhydride (9.50 equiv) in $\mathrm{PhH}(6.25$ $\mathrm{mL})$ at $85{ }^{\circ} \mathrm{C}$ for $30 \mathrm{~min}$, then substrate $(0.250 \mathrm{mmol})$ addition and stirring overnight at $85{ }^{\circ} \mathrm{C}$. ${ }^{b}$ Isolated yields determined after flash column chromatography.

Scheme 1. Preparation of Starting Materials 21 and 25

(a)<smiles>CC1=C2C(=O)C(=O)C=CC2(C)CCC2[C@@H](C)C(=O)O[C@@H]12</smiles>

(b)<smiles>C=C(OCC(=O)C1CCC(C)C(=O)C1)C([18O])Cl</smiles><smiles>CC1=C2C=C(C(C)(O)CO)CCC2(C)C=CC(=O)C1=O</smiles>

23 $\mathrm{h} v, \mathrm{AcOH}$ $72 \%$

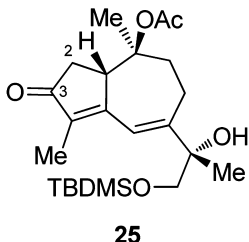
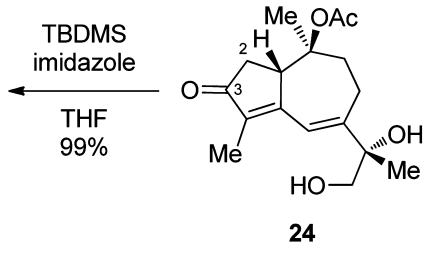

deprotection, affording diol 34 in $61 \%$ yield. Treatment of 34 with $\mathrm{NMO}$ in the presence of catalytic $\mathrm{OsO}_{4}$ produced the dihydroxylation of the $\mathrm{C} 6-\mathrm{C} 7$ double bond, providing tetrol 35 , whose purification resulted to be troublesome. The unpurified tetrol 35 was treated with the TEMPO/NaClO/ $\mathrm{NaClO}_{2}$ oxidation system, leading to guaianolide 36 in $65 \%$ yield (two steps). Guaianolide 36 displays six out of the eight chiral centers found in thapsigargin, thus becoming an interesting model for biological testing.

\section{CONCLUSION}

We have demonstrated that the use of $\mathrm{KMnO}_{4}$ in the presence of a carboxylic acid and its corresponding anhydride is a valid method to perform the introduction of an acyl moiety at the $\alpha^{\prime}$ position of a carbonyl group, enabling the functionalization of hindered enones. The presence of a carboxylic anhydride makes the use of molecular sieves or a Dean-Stark trap to remove the water unnecessary. In addition, the existence of modern statistics software packages makes the DoE analysis available as a tool for the optimization of chemical reactions. The developed conditions have allowed us to prepare highly oxygenated guaianes and guaianolides that can serve as thapsigargin analogues. Further applications to the synthesis of guaianolide derivatives and their biological evaluation are currently in progress.

\section{EXPERIMENTAL SECTION}

General Methods. HPLC purification was carried out using a $1 \times$ $25 \mathrm{~cm}$ silica gel column (LiChrosorb Si 60,7 $\mu \mathrm{m}$ particle size). GC analyses were performed using a DB-5 column. NMR spectra were recorded on a 400 or $500 \mathrm{MHz}$ spectrometer using standard pulse sequences. Spectra were referenced internally to residual solvent signals $\left(\mathrm{CHCl}_{3}, \delta=7.26 \mathrm{ppm}\right.$ for ${ }^{1} \mathrm{H}$ NMR, $\delta=77.16 \mathrm{ppm}$ for ${ }^{13} \mathrm{C}$ NMR). Mass spectra were recorded in a UPLC-QTOF mass spectrometer.

Design of Experiments Optimization (Table 3). All experiments were carried out simultaneously in a parallel synthesis carrousel. In each vessel, $\mathrm{KMnO}_{4}$ (amounts according to Table 3) was vigorously 
Table 6. Scope of the Reaction over Guaianolide and Guaiane Scaffolds ${ }^{a, b}$

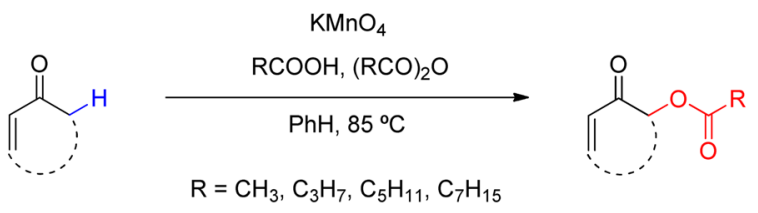<smiles>CC(=O)O[C@H]1C(=O)C(C)=C2[C@H]3OC(=O)C(C)[C@@H]3CCC(C)[C@]21C(C)=O</smiles><smiles>CCCC(=O)O[C@H]1C(=O)C(C)=C2[C@H]3OC(=O)[C@H](C)[C@H]3CCC(C)(C(C)=O)[C@H]21</smiles><smiles>CCCC(=O)O[C@H]1C(=O)C(C)=C2[C@@H]3OC(=O)[C@H](C)[C@H]3CC[C@]1(C)[C@]2(C)C(C)=O</smiles><smiles>CCCC(=O)O[C@H]1C(=O)C(C)=C2[C@@H]3OC(=O)[C@H](C)[C@H]3CCC(C)(C(C)=O)[C@]21C(C)=O</smiles>

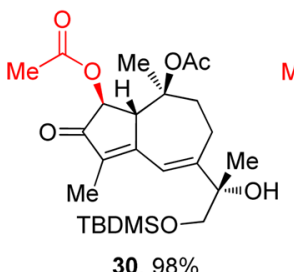

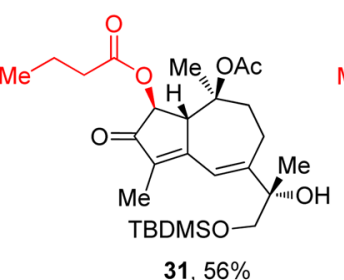

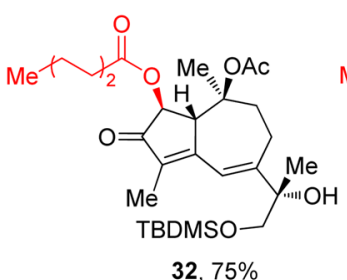

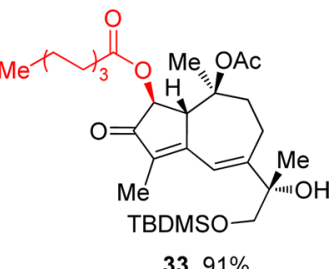

${ }^{a}$ Reaction conditions: $\mathrm{KMnO}_{4}(83 \mathrm{mg}, 0.525 \mathrm{mmol}, 2.10$ equiv), carboxylic acid ( 35.0 equiv), and carboxylic anhydride (9.50 equiv) in $\mathrm{PhH}(6.25$ $\mathrm{mL})$ at $85{ }^{\circ} \mathrm{C}$ for $30 \mathrm{~min}$, then substrate $(0.250 \mathrm{mmol})$ and stir at $85{ }^{\circ} \mathrm{C}$ overnight. ${ }^{b}$ Isolated yields determined after flash column chromatography.

Scheme 2. Synthesis of Compound 36 from 33<smiles>CCCCCCCC(=O)O[C@H]1C(=O)C(C)=C2C=C(C(C)(O)COC(C)(C)C)CC[C@](C)(C(C)=O)[C@H]21</smiles>
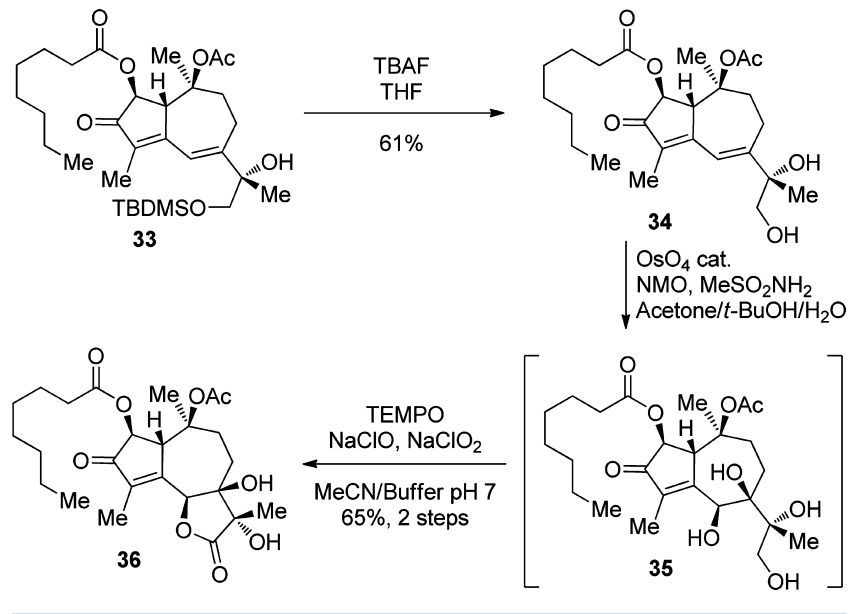

stirred in dry benzene $(3 \mathrm{~mL})$. The mixture was heated at $85{ }^{\circ} \mathrm{C}$, and the corresponding amounts of acetic acid and acetic anhydride were added. After $30 \mathrm{~min}$, the color changed from purple to brown and a solution of isophorone $3(0.025 \mathrm{~mL}, 0.160 \mathrm{mmol})$ and octadecane (GC internal standard, $5 \mathrm{mg}$ ) in dry benzene $(1 \mathrm{~mL}$ ) was added to each vessel. Reactions were vigorously stirred overnight at $85^{\circ} \mathrm{C}$. To determine yields by GC, aliquots from the reaction mixture were taken, filtered through silica gel, and analyzed by GC.

Typical Procedure for $\boldsymbol{\alpha}^{\prime}$-Acyloxylation of Enones (Tables 5 and 6). To a suspension of $\mathrm{KMnO}_{4}(83 \mathrm{mg}, 0.525 \mathrm{mmol}, 2.10$ equiv) in dry benzene $\left(6.25 \mathrm{~mL}, 0.04 \mathrm{M}\right.$ in enone) at $85^{\circ} \mathrm{C}$ were added the corresponding carboxylic acid $(8.750 \mathrm{mmol}, 35.0$ equiv) and its anhydride ( $2.375 \mathrm{mmol}, 9.50$ equiv). The mixture was vigorously stirred until the suspension changed from purple to brown ( $30 \mathrm{~min})$, and then, the enone $(0.250 \mathrm{mmol})$ was added (directly by syringe or dissolved in the minimum amount of dry benzene). Vigorous stirring of the reaction was continued at $85{ }^{\circ} \mathrm{C}$ overnight, then cooled to room temperature, and filtered through an $\mathrm{Al}_{2} \mathrm{O}_{3} /$ Celite pad rinsing with EtOAc. The solvent was evaporated under reduced pressure, and the crude oil was purified by flash column chromatography with EtOAc/ hexanes mixtures as eluents to lead to products in yields according to Tables 5 and 6 .

6-Acetyloxy-3,5,5-trimethylcyclohex-2-enone (4). Yellow oil (49 mg, 100\%); ${ }^{1} \mathrm{H}$ NMR (500 MHz, $\mathrm{CDCl}_{3}$ ) $\delta 5.87$ (br s, $1 \mathrm{H}$ ), 5.18 $(\mathrm{s}, 1 \mathrm{H}), 2.50(\mathrm{~d}, J=18.4 \mathrm{~Hz}, 1 \mathrm{H}), 2.17(\mathrm{~s}, 3 \mathrm{H}), 2.15(\mathrm{~d}, J=16.2 \mathrm{~Hz}$, $1 \mathrm{H}), 1.92(\mathrm{~s}, 3 \mathrm{H}), 1.06(\mathrm{~s}, 3 \mathrm{H}), 0.95(\mathrm{~s}, 3 \mathrm{H}) ;{ }^{13} \mathrm{C}$ NMR $(126 \mathrm{MHz}$, $\left.\mathrm{CDCl}_{3}\right) \delta 193.2$, 170.6, 159.5, 124.7, 80.6, 46.1, 37.7, 27.3, 24.4, 20.8, 20.0; IR (film) $\nu_{\max } 2970,2933,1749,1688,1634,1380,1234,1069$ $\mathrm{cm}^{-1}$; HRMS (APGC) calcd. for $\mathrm{C}_{11} \mathrm{H}_{17} \mathrm{O}_{3}[\mathrm{M}+\mathrm{H}]^{+}$197.1178; found 197.1177.

6-Butanoyloxy-3,5,5-trimethylcyclohex-2-enone (5). Yellow oil (50 mg, 89\%); ${ }^{1} \mathrm{H}$ NMR (400 MHz, $\left.\mathrm{CDCl}_{3}\right) \delta 5.89$ (br s, $\left.1 \mathrm{H}\right), 5.22$ (s, $1 \mathrm{H}), 2.53(\mathrm{~d}, J=18.4 \mathrm{~Hz}, 1 \mathrm{H}), 2.45(\mathrm{~m}, 2 \mathrm{H}), 2.17(\mathrm{~d}, J=18.4 \mathrm{~Hz}$, $1 \mathrm{H}), 1.94(\mathrm{~s}, 3 \mathrm{H}), 1.73(\mathrm{tq}, J=7.4,7.4 \mathrm{~Hz}, 2 \mathrm{H}), 1.08(\mathrm{~s}, 3 \mathrm{H}), 1.00(\mathrm{t}, J$ $=7.4 \mathrm{~Hz}, 3 \mathrm{H}), 0.97(\mathrm{~s}, 3 \mathrm{H}) ;{ }^{13} \mathrm{C} \mathrm{NMR}\left(100 \mathrm{MHz}, \mathrm{CDCl}_{3}\right) \delta 193.4$, 173.3, 159.3, 124.9, 80.4, 46.2, 37.9, 36.2, 27.4, 24.4, 20.1, 18.9, 13.8; IR (film) $\nu_{\max } 2968,2937,2876,1743,1688,1636,1381,1238,1176$, $1094 \mathrm{~cm}^{-1}$; HRMS (APGC) calcd. for $\mathrm{C}_{13} \mathrm{H}_{20} \mathrm{O}_{3}[\mathrm{M}]^{+}$224.1412; found 224.1425 .

6-Hexanoyloxy-3,5,5-trimethylcyclohex-2-enone (6). Yellow oil (51 mg, 81\%); ${ }^{1} \mathrm{H}$ NMR (400 MHz, $\left.\mathrm{CDCl}_{3}\right) \delta 5.88$ (br s, $\left.1 \mathrm{H}\right), 5.20$ (s, $1 \mathrm{H}), 2.51(\mathrm{~d}, J=19.1 \mathrm{~Hz}, 1 \mathrm{H}), 2.45(\mathrm{~m}, 2 \mathrm{H}), 2.16(\mathrm{~d}, J=18.4 \mathrm{~Hz}$, $1 \mathrm{H}), 1.93(\mathrm{~s}, 3 \mathrm{H}), 1.64(\mathrm{tt}, J=7.5,7.4 \mathrm{~Hz}, 2 \mathrm{H}), 1.34(\mathrm{~m}, 4 \mathrm{H}), 1.06(\mathrm{~s}$, $3 \mathrm{H}), 0.96(\mathrm{~s}, 3 \mathrm{H}), 0.88(\mathrm{t}, J=7.1 \mathrm{~Hz}, 3 \mathrm{H}) ;{ }^{13} \mathrm{C}$ NMR $(100 \mathrm{MHz}$, $\left.\mathrm{CDCl}_{3}\right) \delta 193.3,173.4,159.3,124.8,80.3,46.2,37.8,34.2,31.4,27.4$, 24.8, 24.4, 22.4, 20.1, 14.0; IR (film) $\nu_{\max } 2961,2934,2873,1744$, 1690, 1634, 1380, 1238, 1169, $1059 \mathrm{~cm}^{-1}$; HRMS (APGC) calcd. for $\mathrm{C}_{15} \mathrm{H}_{25} \mathrm{O}_{3}[\mathrm{M}+\mathrm{H}]^{+}$253.1804; found 253.1808.

3,5,5-Trimethyl-6-octanoyloxycyclohex-2-enone (7). Yellow oil (46 mg, 66\%); ${ }^{1} \mathrm{H}$ NMR (400 MHz, $\left.\mathrm{CDCl}_{3}\right) \delta 5.89$ (br s, $\left.1 \mathrm{H}\right), 5.21$ (s, $1 \mathrm{H}), 2.52(\mathrm{~d}, J=18.4 \mathrm{~Hz}, 1 \mathrm{H}), 2.45(\mathrm{~m}, 2 \mathrm{H}), 2.16(\mathrm{~d}, J=18.4 \mathrm{~Hz}$, $1 \mathrm{H}), 1.93(\mathrm{~s}, 3 \mathrm{H}), 1.68(\mathrm{tt}, J=7.5,7.4 \mathrm{~Hz}, 2 \mathrm{H}), 1.31(\mathrm{~m}, 8 \mathrm{H}), 1.07(\mathrm{~s}$, $3 \mathrm{H}), 0.97(\mathrm{~s}, 3 \mathrm{H}), 0.86(\mathrm{t}, J=6.9 \mathrm{~Hz}, 3 \mathrm{H}) ;{ }^{13} \mathrm{C}$ NMR $(100 \mathrm{MHz}$, $\left.\mathrm{CDCl}_{3}\right) \delta 193.3,173.4,159.3,124.9,80.3,46.2,37.8,34.3,31.8,29.2$, 29.1, 27.4, 25.2, 24.4, 22.7, 20.1, 14.2; IR (film) $\nu_{\max } 2958,2931,2858$, $1745,1690,1637,1380,1165,1065 \mathrm{~cm}^{-1}$; HRMS (APGC) calcd. for $\mathrm{C}_{17} \mathrm{H}_{29} \mathrm{O}_{3}[\mathrm{M}+\mathrm{H}]^{+}$281.2117; found 281.2108.

6-Acetyloxy-3-methylcyclohex-2-enone (8). Yellow oil (30 mg, $71 \%) ;{ }^{1} \mathrm{H}$ NMR $\left(500 \mathrm{MHz}, \mathrm{CDCl}_{3}\right) \delta 5.90(\mathrm{br} \mathrm{s}, 1 \mathrm{H}), 5.29(\mathrm{br} \mathrm{s}, 1 \mathrm{H})$, $2.55(\mathrm{~m}, 1 \mathrm{H}), 2.39(\mathrm{br} \mathrm{d}, J=18.7 \mathrm{~Hz}, 1 \mathrm{H}), 2.23(\mathrm{~m}, 1 \mathrm{H}), 2.16(\mathrm{~s}, 3 \mathrm{H})$, $2.09(\mathrm{~m}, 1 \mathrm{H}), 1.96(\mathrm{~s}, 3 \mathrm{H}) ;{ }^{13} \mathrm{C} \mathrm{NMR}\left(126 \mathrm{MHz}, \mathrm{CDCl}_{3}\right) \delta 193.6$, 
170.4, 162.4, 125.5, 73.2, 30.5, 28.4, 24.3, 21.0; IR (film) $\nu_{\max } 2936$, 1747, 1690, 1380, 1238, 1208, $1168 \mathrm{~cm}^{-1}$; HRMS (APGC) calcd. for $\mathrm{C}_{9} \mathrm{H}_{13} \mathrm{O}_{3}[\mathrm{M}+\mathrm{H}]^{+}$169.0865; found 169.0868 .

6-Butanoyloxy-3-methylcyclohex-2-enone (9). Yellow oil (27 $\mathrm{mg}, 56 \%) ;{ }^{1} \mathrm{H}$ NMR $\left(500 \mathrm{MHz}, \mathrm{CDCl}_{3}\right) \delta 5.90(\mathrm{br} \mathrm{s}, 1 \mathrm{H}), 5.31$ (dd, $J$ $=13.3,5.3 \mathrm{~Hz}, 1 \mathrm{H}), 2.55(\mathrm{~m}, 1 \mathrm{H}), 2.40(\mathrm{~m}, 3 \mathrm{H}), 2.22(\mathrm{dddd}, J=7.8$, 7.8, 5.2, $2.6 \mathrm{~Hz}, 1 \mathrm{H}$ ), 2.09 (dddd, $J=17.1,12.7,12.7,5.2 \mathrm{~Hz}, 1 \mathrm{H}$ ), $1.97(\mathrm{~s}, 3 \mathrm{H}), 1.70(\mathrm{tq}, J=7.4,7.4 \mathrm{~Hz}, 2 \mathrm{H}), 0.97(\mathrm{t}, J=7.4 \mathrm{~Hz}, 3 \mathrm{H})$; ${ }^{13} \mathrm{C} \mathrm{NMR}\left(126 \mathrm{MHz}, \mathrm{CDCl}_{3}\right) \delta 193.7,173.0,162.3,125.6,72.9,36.2$, 30.6, 28.4, 24.3, 18.6, 13.7; IR (film) $\nu_{\max } 2966,2936,2876,1743$, $1689,1633,1381,1177,1104 \mathrm{~cm}^{-1}$; HRMS (APGC) calcd. for $\mathrm{C}_{11} \mathrm{H}_{17} \mathrm{O}_{3}[\mathrm{M}+\mathrm{H}]^{+}$197.1178; found 197.1178.

6-Hexanoyloxy-3-methylcyclohex-2-enone (10). Yellow oil (37 mg, 66\%); ${ }^{1} \mathrm{H}$ NMR (400 MHz, $\left.\mathrm{CDCl}_{3}\right) \delta 5.89$ (br s, $\left.1 \mathrm{H}\right)$, $5.30(\mathrm{dd}, J=13.3,5.4 \mathrm{~Hz}, 1 \mathrm{H}), 2.55(\mathrm{~m}, 1 \mathrm{H}), 2.40(\mathrm{~m}, 3 \mathrm{H}), 2.21$ (dddd, $J=7.8,7.8,5.2,2.6 \mathrm{~Hz}, 1 \mathrm{H}$ ), 2.08 (dddd, $J=16.9,13.2,11.7$, $5.2 \mathrm{~Hz}, 1 \mathrm{H}), 1.96(\mathrm{~s}, 3 \mathrm{H}), 1.66(\mathrm{tt}, J=7.7,7.7 \mathrm{~Hz}, 2 \mathrm{H}), 1.33(\mathrm{~m}, 4 \mathrm{H})$, $0.88(\mathrm{t}, J=7.1 \mathrm{~Hz}, 3 \mathrm{H}) ;{ }^{13} \mathrm{C}$ NMR $\left(100 \mathrm{MHz}, \mathrm{CDCl}_{3}\right) \delta 193.7,173.2$, 162.3, 125.6, 72.9, 34.3, 31.4, 30.5, 28.4, 24.7, 24.2, 22.4, 14.0; IR (film) $\nu_{\max } 2956,2933,2872,1743,1690,1633,1380,1208,1167$, $1103 \mathrm{~cm}^{-1}$; HRMS (APGC) calcd. for $\mathrm{C}_{13} \mathrm{H}_{21} \mathrm{O}_{3}[\mathrm{M}+\mathrm{H}]^{+}$225.1491; found 225.1505.

3-Methyl-6-octanoyloxycyclohex-2-enone (11). Yellow oil (47 $\mathrm{mg}, 74 \%) ;{ }^{1} \mathrm{H}$ NMR (400 MHz, $\left.\mathrm{CDCl}_{3}\right) \delta 5.90($ br s, $1 \mathrm{H}), 5.31$ (dd, $J$ $=13.3,5.4 \mathrm{~Hz}, 1 \mathrm{H}), 2.55(\mathrm{~m}, 1 \mathrm{H}), 2.39(\mathrm{~m}, 3 \mathrm{H}), 2.22(\mathrm{dddd}, J=7.8$, 7.8, 5.2, $2.6 \mathrm{~Hz}, 1 \mathrm{H}), 2.09$ (dddd, $J=16.9,11.8,11.8,5.2 \mathrm{~Hz}, 1 \mathrm{H}$ ), $1.97(\mathrm{~s}, 3 \mathrm{H}), 1.67(\mathrm{tt}, J=7.1,7.1 \mathrm{~Hz}, 2 \mathrm{H}), 1.31(\mathrm{~m}, 8 \mathrm{H}), 0.87(\mathrm{t}, J=$ $6.8 \mathrm{~Hz}, 3 \mathrm{H}) ;{ }^{13} \mathrm{C}$ NMR $\left(100 \mathrm{MHz}, \mathrm{CDCl}_{3}\right) \delta 193.7,173.2,162.3$, 125.6, 72.9, 34.3, 31.8, 30.6, 29.2, 29.1, 28.4, 25.0, 24.3, 22.7, 14.2; IR (film) $\nu_{\max } 2930,2856,1743,1691,1633,1380,1162,1105 \mathrm{~cm}^{-1}$; HRMS (APGC) calcd. for $\mathrm{C}_{15} \mathrm{H}_{25} \mathrm{O}_{3}[\mathrm{M}+\mathrm{H}]^{+}$253.1804; found 253.1797.

5-Acetyloxy-3-methyl-2-pentylcyclopent-2-enone (12). Yellow oil (45 mg, 81\%); ${ }^{1} \mathrm{H}$ NMR (400 MHz, $\left.\mathrm{CDCl}_{3}\right) \delta 5.11$ (dd, $J=$ 6.9, $3.0 \mathrm{~Hz}, 1 \mathrm{H}), 2.97(\mathrm{dd}, J=18.0,6.9 \mathrm{~Hz}, 1 \mathrm{H}), 2.42(\mathrm{br} \mathrm{d}, J=18.0$ $\mathrm{Hz}, 1 \mathrm{H}), 2.18(\mathrm{~m}, 2 \mathrm{H}), 2.12(\mathrm{~s}, 3 \mathrm{H}), 2.05(\mathrm{~s}, 3 \mathrm{H}), 1.38(\mathrm{~m}, 2 \mathrm{H}), 1.28$ $(\mathrm{m}, 4 \mathrm{H}), 0.87(\mathrm{t}, J=7.1 \mathrm{~Hz}, 3 \mathrm{H}) ;{ }^{13} \mathrm{C}$ NMR $\left(100 \mathrm{MHz}, \mathrm{CDCl}_{3}\right) \delta$ 203.3, 170.8, 167.4, 139.6, 71.9, 38.9, 31.8, 28.0, 23.1, 22.6, 21.0, 17.3, 14.1; IR (film) $\nu_{\max } 2931,2860,1746,1714,1373,1229,1056 \mathrm{~cm}^{-1}$; HRMS (APGC) calcd. for $\mathrm{C}_{13} \mathrm{H}_{21} \mathrm{O}_{3}[\mathrm{M}+\mathrm{H}]^{+}$225.1491; found 225.1513.

5-Butanoyloxy-3-methyl-2-pentylcyclopent-2-enone (13). Yellow oil (22 mg, 35\%); ${ }^{1} \mathrm{H}$ NMR (500 MHz, $\left.\mathrm{CDCl}_{3}\right) \delta 5.12$ (dd, $J=6.9,3.0 \mathrm{~Hz}, 1 \mathrm{H}), 2.97(\mathrm{dd}, J=18.0,6.9 \mathrm{~Hz}, 1 \mathrm{H}), 2.36(\mathrm{~m}, 3 \mathrm{H})$, $2.18(\mathrm{~m}, 2 \mathrm{H}), 2.05(\mathrm{~s}, 3 \mathrm{H}), 1.67(\mathrm{tq}, J=7.4,7.1 \mathrm{~Hz}, 2 \mathrm{H}), 1.39(\mathrm{tt}, J=$ $7.8,7.8 \mathrm{~Hz}, 2 \mathrm{H}), 1.27(\mathrm{~m}, 4 \mathrm{H}), 0.96(\mathrm{t}, J=7.4 \mathrm{~Hz}, 3 \mathrm{H}), 0.86(\mathrm{t}, J=7.1$ $\mathrm{Hz}, 3 \mathrm{H}) ;{ }^{13} \mathrm{C}$ NMR $\left(126 \mathrm{MHz}, \mathrm{CDCl}_{3}\right) \delta 203.3,173.4,167.2,139.6$, 71.8, 38.9, 36.0, 31.8, 28.0, 23.1, 22.6, 18.5, 17.3, 14.1, 13.7; IR (film) $\nu_{\max } 2959,2932,2874,1743,1714,1643,1388,1174,1055 \mathrm{~cm}^{-1}$; HRMS (APGC) calcd. for $\mathrm{C}_{15} \mathrm{H}_{25} \mathrm{O}_{3}[\mathrm{M}+\mathrm{H}]^{+}$253.1804; found 253.1844 .

5-Hexanoyloxy-3-methyl-2-pentylcyclopent-2-enone (14). Yellow oil (52 mg, 74\%); ${ }^{1} \mathrm{H}$ NMR (400 MHz, $\left.\mathrm{CDCl}_{3}\right) \delta 5.11$ (dd, $J=6.9,3.1 \mathrm{~Hz}, 1 \mathrm{H}), 2.96(\mathrm{dd}, J=18.0,6.9 \mathrm{~Hz}, 1 \mathrm{H}), 2.36(\mathrm{~m}, 3 \mathrm{H})$, $2.18(\mathrm{~m}, 2 \mathrm{H}), 2.05(\mathrm{~s}, 3 \mathrm{H}), 1.64(\mathrm{tt}, J=7.5,7.5 \mathrm{~Hz}, 2 \mathrm{H}), 1.38(\mathrm{~m}$, $2 \mathrm{H}), 1.27(\mathrm{~m}, 8 \mathrm{H}), 0.88(\mathrm{~m}, 6 \mathrm{H}) ;{ }^{13} \mathrm{C} \mathrm{NMR}\left(100 \mathrm{MHz}, \mathrm{CDCl}_{3}\right) \delta$ 203.3, 173.6, 167.1, 139.6, 71.8, 38.9, 34.2, 31.8, 31.4, 28.0, 24.7, 23.1, 22.6, 22.4, 17.3, 14.1, 14.0; IR (film) $\nu_{\max } 2956,2931,2859,1743$, $1715,1643,1388,1164,1112 \mathrm{~cm}^{-1}$; HRMS (APGC) calcd. for $\mathrm{C}_{17} \mathrm{H}_{29} \mathrm{O}_{3}[\mathrm{M}+\mathrm{H}]^{+}$281.2117; found 281.2126.

3-Methyl-5-octanoyloxy-2-pentylcyclopent-2-enone (15). Yellow oil (42 mg, 54\%); ${ }^{1} \mathrm{H}$ NMR $\left(500 \mathrm{MHz}, \mathrm{CDCl}_{3}\right) \delta 5.11(\mathrm{dd}$, $J=6.9,3.0 \mathrm{~Hz}, 1 \mathrm{H}), 2.96(\mathrm{dd}, J=18.0,6.9 \mathrm{~Hz}, 1 \mathrm{H}), 2.36(\mathrm{~m}, 3 \mathrm{H})$, $2.18(\mathrm{~m}, 2 \mathrm{H}), 2.04(\mathrm{~s}, 3 \mathrm{H}), 1.63(\mathrm{tt}, J=7.8,7.8 \mathrm{~Hz}, 2 \mathrm{H}), 1.38(\mathrm{tt}, J=$ 7.6, 7.6 Hz, 2H), $1.26(\mathrm{~m}, 12 \mathrm{H}), 0.86(\mathrm{~m}, 6 \mathrm{H}) ;{ }^{13} \mathrm{C} \mathrm{NMR}(126 \mathrm{MHz}$, $\left.\mathrm{CDCl}_{3}\right) \delta 203.3,173.6,167.1,139.6,71.7,38.9,34.2,31.8,31.7,29.2$, 29.0, 28.0, 25.0, 23.1, 22.7, 22.6, 17.3, 14.2, 14.1; IR (film) $\nu_{\max } 2956$, 2929, 2858, 1745, 1716,1645, 1387, 1162, $1110 \mathrm{~cm}^{-1}$; HRMS (APGC) calcd. for $\mathrm{C}_{19} \mathrm{H}_{33} \mathrm{O}_{3}[\mathrm{M}+\mathrm{H}]^{+}$309.2430; found 309.2458.
6-Acetyloxy-3-ethoxycyclohex-2-enone (16). Yellow oil (29 $\mathrm{mg}, 59 \%) ;{ }^{1} \mathrm{H} \mathrm{NMR}\left(400 \mathrm{MHz}, \mathrm{CDCl}_{3}\right) \delta 5.36(\mathrm{~s}, 1 \mathrm{H}), 5.28$ (dd, $J=$ 12.6, $5.2 \mathrm{~Hz}, 1 \mathrm{H}), 3.90(\mathrm{~m}, 2 \mathrm{H}), 2.64$ (ddd, $\mathrm{J}=17.1,12.0,5.1 \mathrm{~Hz}$, $1 \mathrm{H}$ ), 2.49 (ddd, $J=17.8,5.3,2.9 \mathrm{~Hz}, 1 \mathrm{H}), 2.21$ (dddd, $J=10.4,5.2$, 4.6, $3.0 \mathrm{~Hz}, 1 \mathrm{H}), 2.16(\mathrm{~s}, 3 \mathrm{H}), 2.08$ (dddd, $J=17.6,12.5,12.5,5.3 \mathrm{~Hz}$, $1 \mathrm{H}), 1.36(\mathrm{t}, J=7.0 \mathrm{~Hz}, 3 \mathrm{H}) ;{ }^{13} \mathrm{C} \mathrm{NMR}\left(100 \mathrm{MHz}, \mathrm{CDCl}_{3}\right) \delta 193.3$, 176.8, 170.5, 101.4, 72.6, 65.0, 28.1, 26.9, 21.1, 14.2; IR (film) $\nu_{\max }$ 2984, 1741, 1673,1602,1378, 1236, 1191, $1046 \mathrm{~cm}^{-1}$; HRMS (APGC) calcd. for $\mathrm{C}_{10} \mathrm{H}_{15} \mathrm{O}_{4}[\mathrm{M}+\mathrm{H}]^{+}$199.0970; found 199.0986 .

6-Butanoyloxy-3-ethoxycyclohex-2-enone (17). Yellow oil (48 $\mathrm{mg}, 85 \%) ;{ }^{1} \mathrm{H} \mathrm{NMR}\left(400 \mathrm{MHz}, \mathrm{CDCl}_{3}\right) \delta 5.33(\mathrm{~s}, 1 \mathrm{H}), 5.27$ (dd, $J=$ $12.5,5.3 \mathrm{~Hz}, 1 \mathrm{H}), 3.88(\mathrm{~m}, 2 \mathrm{H}), 2.63$ (ddd, $J=17.4,11.9,5.2 \mathrm{~Hz}$, $1 \mathrm{H}), 2.47(\mathrm{ddd}, J=17.8,5.3,3.2 \mathrm{~Hz}, 1 \mathrm{H}), 2.37(\mathrm{~m}, 2 \mathrm{H}), 2.18$ (dddd, $J$ $=8.3,8.3,5.2,3.0 \mathrm{~Hz}, 1 \mathrm{H}), 2.06$ (dddd, $J=17.7,12.4,12.4,5.3 \mathrm{~Hz}$, $1 \mathrm{H}), 1.68(\mathrm{tq}, J=7.4,7.0 \mathrm{~Hz}, 2 \mathrm{H}), 1.34(\mathrm{t}, J=7.0 \mathrm{~Hz}, 3 \mathrm{H}), 0.95(\mathrm{t}, J=$ $7.4 \mathrm{~Hz}, 3 \mathrm{H}) ;{ }^{13} \mathrm{C}$ NMR $\left(100 \mathrm{MHz}, \mathrm{CDCl}_{3}\right) \delta 193.3,176.6,173.0$, 101.4, 72.3, 64.9, 36.1, 28.1, 26.9, 18.5, 14.1, 13.7; IR (film) $\nu_{\max } 2966$, 2940, 2877, 1743, 1678,1605, 1380, 1187, $1101 \mathrm{~cm}^{-1}$; HRMS (APGC) calcd. for $\mathrm{C}_{12} \mathrm{H}_{19} \mathrm{O}_{4}[\mathrm{M}+\mathrm{H}]^{+}$227.1283; found 227.1295.

3-Ethoxy-6-hexanoyloxycyclohex-2-enone (18). Yellow oil (51 mg, 81\%); ${ }^{1} \mathrm{H}$ NMR (500 MHz, $\left.\mathrm{CDCl}_{3}\right) \delta 5.35$ (s, 1H), 5.27 (dd, $J=12.6,5.2 \mathrm{~Hz}, 1 \mathrm{H}), 3.89(\mathrm{~m}, 2 \mathrm{H}), 2.63(\mathrm{ddd}, J=17.5,12.0,5.1$, $\mathrm{Hz}, 1 \mathrm{H}), 2.48$ (ddd, $J=17.8,5.2,2.9 \mathrm{~Hz}, 1 \mathrm{H}), 2.39$ (m, $2 \mathrm{H}), 2.19$ (dddd, $J=8.2,8.2,5.2,3.0 \mathrm{~Hz}, 1 \mathrm{H}$ ), 2.07 (dddd, $J=17.8,12.5,12.5$, $5.3 \mathrm{~Hz}, 1 \mathrm{H}), 1.66(\mathrm{tt}, J=7.6,7.6 \mathrm{~Hz}, 2 \mathrm{H}), 1.34(\mathrm{t}, J=7.1 \mathrm{~Hz}, 3 \mathrm{H})$, $1.31(\mathrm{~m}, 4 \mathrm{H}), 0.87(\mathrm{t}, J=7.1 \mathrm{~Hz}, 3 \mathrm{H}) ;{ }^{13} \mathrm{C} \mathrm{NMR}\left(126 \mathrm{MHz}, \mathrm{CDCl}_{3}\right)$ $\delta$ 193.3, 176.7, 173.2, 101.4, 72.3, 65.0, 34.3, 31.3, 28.1, 26.9, 24.7, 22.4, 14.2, 14.0; IR (film) $\nu_{\max } 2954,2933,2872,1744,1667,1604$, $1379,1196,1169 \mathrm{~cm}^{-1}$; HRMS (APGC) calcd. for $\mathrm{C}_{14} \mathrm{H}_{23} \mathrm{O}_{4}[\mathrm{M}+$ $\mathrm{H}]^{+}$255.1596; found 255.1596.

3-Ethoxy-6-octanoyloxycyclohex-2-enone (19). Yellow oil (61 $\mathrm{mg}, 86 \%) ;{ }^{1} \mathrm{H}$ NMR (400 MHz, $\left.\mathrm{CDCl}_{3}\right) \delta 5.35(\mathrm{~s}, 1 \mathrm{H}), 5.27$ (dd, $J=$ 12.5, $5.2 \mathrm{~Hz}, 1 \mathrm{H}), 3.88(\mathrm{~m}, 2 \mathrm{H}), 2.63(\mathrm{ddd}, J=17.4,11.9,5.0 \mathrm{~Hz}$, $1 \mathrm{H}), 2.48$ (ddd, $J=17.6,5.1,2.8 \mathrm{~Hz}, 1 \mathrm{H}), 2.40$ (m, 2H), 2.19 (dddd, $J$ $=8.3,8.3,5.2,3.0 \mathrm{~Hz}, 1 \mathrm{H}), 2.07$ (dddd, $J=17.8,12.3,12.3,5.3 \mathrm{~Hz}$, $1 \mathrm{H}), 1.65(\mathrm{tt}, J=7.2,7.4 \mathrm{~Hz}, 2 \mathrm{H}), 1.34(\mathrm{t}, J=7.0 \mathrm{~Hz}, 3 \mathrm{H}), 1.28(\mathrm{~m}$, $8 \mathrm{H}), 0.85(\mathrm{t}, J=6.9 \mathrm{~Hz}, 3 \mathrm{H}) ;{ }^{13} \mathrm{C} \mathrm{NMR}\left(100 \mathrm{MHz}, \mathrm{CDCl}_{3}\right) \delta 193.3$, 176.7, 173.2, 101.4, 72.3, 64.9, 34.3, 31.7, 29.1, 29.0, 28.1, 26.9, 25.0, 22.7, 14.2, 14.1; IR (film) $\nu_{\max } 2951,2917,2852,1744,1661,1605$, 1470, 1170, $872 \mathrm{~cm}^{-1}$; HRMS (APGC) calcd. for $\mathrm{C}_{16} \mathrm{H}_{27} \mathrm{O}_{4}[\mathrm{M}+\mathrm{H}]^{+}$ 283.1909; found 283.1925.

(3S,3aS,6R,6aS,7R,9bS)-7-Acetyloxy-3,6,9-trimethyl-2,8dioxo-2,3,3a,4,5,6,6a,7,8,9b-decahydroazuleno[4,5-b]furan-6yl Acetate (26). White amorphous solid $(55 \mathrm{mg}, 60 \%) ;[\alpha]_{\mathrm{D}}^{20}+45.33$ (c 1.16, $\left.\mathrm{CHCl}_{3}\right) ;{ }^{1} \mathrm{H}$ NMR $\left(500 \mathrm{MHz}, \mathrm{CDCl}_{3}\right) \delta 5.13(\mathrm{~d}, J=3.4 \mathrm{~Hz}$, $1 \mathrm{H}), 4.83$ (br d, $J=11.0 \mathrm{~Hz}, 1 \mathrm{H}), 4.05$ (br s, $1 \mathrm{H}), 2.60$ (ddd, $J=18.1$, 13.6, $4.4 \mathrm{~Hz}, 1 \mathrm{H}), 2.28(\mathrm{~m}, 3 \mathrm{H}), 2.14(\mathrm{~m}, 1 \mathrm{H}), 2.09(\mathrm{~s}, 3 \mathrm{H}), 2.06(\mathrm{~m}$, $1 \mathrm{H}), 1.95(\mathrm{~s}, 3 \mathrm{H}), 1.95$ (s, 3H), 1.46 (dddd, $J=14.8,13.6,11.3,3.7$ $\mathrm{Hz}, 1 \mathrm{H}), 1.27(\mathrm{~d}, J=6.9 \mathrm{~Hz}, 3 \mathrm{H}), 1.24(\mathrm{~s}, 3 \mathrm{H}) ;{ }^{13} \mathrm{C} \mathrm{NMR}(126 \mathrm{MHz}$, $\left.\mathrm{CDCl}_{3}\right) \delta 201.1,176.7,170.5,169.8,157.7,140.6,84.7,81.1,73.7$, $52.5,48.6,41.3,37.9,25.7,22.6,20.8,20.7,12.6,10.1$; IR (film) $\nu_{\max }$ 2931, 1784, 1728, 1235, 1017, $753 \mathrm{~cm}^{-1}$; HRMS (APGC) calcd. for $\mathrm{C}_{19} \mathrm{H}_{25} \mathrm{O}_{7}[\mathrm{M}+\mathrm{H}]^{+}$365.1600; found 365.1612 .

(3S,3aS,6R,6aS,7R,9bS)-7-Butanoyloxy-3,6,9-trimethyl-2,8dioxo-2,3,3a,4,5,6,6a,7,8,9b-decahydroazuleno[4,5-b]furan-6yl Acetate (27). White amorphous solid $(60 \mathrm{mg}, 61 \%) ;[\alpha]_{\mathrm{D}}^{20}+90.95$ $\left(\right.$ c 2.86, $\left.\mathrm{CHCl}_{3}\right) ;{ }^{1} \mathrm{H}$ NMR $\left(400 \mathrm{MHz}, \mathrm{CDCl}_{3}\right) \delta 5.11(\mathrm{~d}, J=3.2 \mathrm{~Hz}$, $1 \mathrm{H}), 4.82$ (br d, $J=11.0 \mathrm{~Hz}, 1 \mathrm{H}$ ), 4.04 (br s, $1 \mathrm{H}), 2.59$ (ddd, $J=18.0$, 13.6, $4.4 \mathrm{~Hz}, 1 \mathrm{H}), 2.30(\mathrm{~m}, 3 \mathrm{H}), 2.23(\mathrm{t}, J=3.6 \mathrm{~Hz}, 1 \mathrm{H}), 2.15(\mathrm{~m}$, $1 \mathrm{H}), 2.06(\mathrm{~m}, 1 \mathrm{H}), 1.94(\mathrm{~s}, 3 \mathrm{H}), 1.93(\mathrm{~s}, 3 \mathrm{H}), 1.64(\mathrm{tq}, J=7.5,7.5 \mathrm{~Hz}$, $2 \mathrm{H}), 1.45$ (dddd, $J=14.6,14.6,11.2,3.6 \mathrm{~Hz}, 1 \mathrm{H}), 1.25(\mathrm{~d}, J=6.9 \mathrm{~Hz}$, $3 \mathrm{H}), 1.23(\mathrm{~s}, 3 \mathrm{H}), 0.96(\mathrm{t}, J=7.4 \mathrm{~Hz}, 3 \mathrm{H}) ;{ }^{13} \mathrm{C} \mathrm{NMR}(100 \mathrm{MHz}$, $\left.\mathrm{CDCl}_{3}\right) \delta 201.2,176.7,172.5,170.5,157.8,140.6,84.6,81.1,73.6$, $52.5,48.5,41.3,37.9,35.7,25.6,22.6,20.8,18.3,13.7,12.6,10.1$; IR (film) $\nu_{\max } 2967,2876,1785,1724,1250,1171,997 \mathrm{~cm}^{-1}$; HRMS (APGC) calcd. for $\mathrm{C}_{21} \mathrm{H}_{29} \mathrm{O}_{7}[\mathrm{M}+\mathrm{H}]^{+}$393.1913; found 393.1904.

(3S,3aS,6R,6aS,7R,9bS)-7-Hexanoyloxy-3,6,9-trimethyl-2,8dioxo-2,3,3a,4,5,6,6a,7,8,9b-decahydroazuleno[4,5- $b$ ]furan-6yl Acetate (28). White amorphous solid $(67 \mathrm{mg}, 64 \%) ;[\alpha]_{\mathrm{D}}^{20}+50.97$ (c 0.42, $\left.\mathrm{CHCl}_{3}\right) ;{ }^{1} \mathrm{H}$ NMR $\left(400 \mathrm{MHz}, \mathrm{CDCl}_{3}\right) \delta 5.10(\mathrm{~d}, J=3.1 \mathrm{~Hz}$, 
$1 \mathrm{H}), 4.82(\mathrm{br} \mathrm{d}, J=11.0 \mathrm{~Hz}, 1 \mathrm{H}), 4.05$ (br s, $1 \mathrm{H}), 2.60$ (ddd, $J=18.0$, 13.6, $4.4 \mathrm{~Hz}, 1 \mathrm{H}), 2.31(\mathrm{~m}, 3 \mathrm{H}), 2.25(\mathrm{~m}, 1 \mathrm{H}), 2.15(\mathrm{~m}, 1 \mathrm{H}), 2.06(\mathrm{~m}$, $1 \mathrm{H}), 1.94(\mathrm{~s}, 3 \mathrm{H}), 1.93(\mathrm{~s}, 3 \mathrm{H}), 1.61(\mathrm{tt}, J=7.1,7.1 \mathrm{~Hz}, 2 \mathrm{H}), 1.45$ (dddd, $J=14.6,14.6,11.2,3.6 \mathrm{~Hz}, 1 \mathrm{H}), 1.31(\mathrm{~m}, 4 \mathrm{H}), 1.25(\mathrm{~d}, J=6.9$ $\mathrm{Hz}, 3 \mathrm{H}), 1.23(\mathrm{~s}, 3 \mathrm{H}), 0.87(\mathrm{t}, J=7.1 \mathrm{~Hz}, 3 \mathrm{H}) ;{ }^{13} \mathrm{C} \mathrm{NMR}(100 \mathrm{MHz}$, $\left.\mathrm{CDCl}_{3}\right) \delta 201.2,176.7,172.6,170.5,157.7,140.6,84.6,81.1,73.6$, 52.5, 48.5, 41.3, 37.9, 33.8, 31.2, 25.7, 24.4, 22.6, 22.4, 20.9, 14.0, 12.6, 10.1; IR (film) $\nu_{\max } 2935,2873,1787,1727,1249,1170,998 \mathrm{~cm}^{-1}$; HRMS (APGC) calcd. for $\mathrm{C}_{23} \mathrm{H}_{33} \mathrm{O}_{7}[\mathrm{M}+\mathrm{H}]^{+}$421.2226; found 421.2214 .

(3S,3aS,6R,6aS,7R,9bS)-3,6,9-Trimethyl-7-octanoyloxy-2,8dioxo-2,3,3a,4,5,6,6a,7,8,9b-decahydroazuleno[4,5-b]furan-6yl Acetate (29). White amorphous solid (80 mg, 71\%); $[\alpha]_{\mathrm{D}}^{20}+44.34$ (c 0.99, $\left.\mathrm{CHCl}_{3}\right) ;{ }^{1} \mathrm{H}$ NMR $\left(500 \mathrm{MHz}, \mathrm{CDCl}_{3}\right) \delta 5.13(\mathrm{~d}, J=3.3 \mathrm{~Hz}$, $1 \mathrm{H}), 4.82$ (br d, $J=11.2 \mathrm{~Hz}, 1 \mathrm{H}), 4.06$ (br s, $1 \mathrm{H}), 2.61$ (ddd, $J=18.1$, 13.6, $4.4 \mathrm{~Hz}, 1 \mathrm{H}), 2.34(\mathrm{~m}, 3 \mathrm{H}), 2.29(\mathrm{~m}, 1 \mathrm{H}), 2.25(\mathrm{~m}, 1 \mathrm{H}), 2.15(\mathrm{~m}$, $1 \mathrm{H}), 2.08(\mathrm{~m}, 1 \mathrm{H}), 1.96(\mathrm{~s}, 6 \mathrm{H}), 1.62(\mathrm{tt}, J=7.1,7.1 \mathrm{~Hz}, 2 \mathrm{H}), 1.46$ (dddd, $J=14.7,14.7,11.2,3.6 \mathrm{~Hz}, 1 \mathrm{H}), 1.32(\mathrm{~m}, 8 \mathrm{H}), 1.28(\mathrm{~d}, J=6.9$ $\mathrm{Hz}, 3 \mathrm{H}), 1.24(\mathrm{~s}, 3 \mathrm{H}), 0.87(\mathrm{t}, J=7.0 \mathrm{~Hz}, 3 \mathrm{H}) ;{ }^{13} \mathrm{C} \mathrm{NMR}(126 \mathrm{MHz}$, $\left.\mathrm{CDCl}_{3}\right) \delta 201.2,176.8,172.7,170.5,157.7,140.7,84.7,81.2,73.6$, 52.6, 48.6, 41.4, 37.9, 33.9, 31.8, 29.1, 29.1, 25.7, 24.8, 22.7, 22.6, 20.9, 14.2, 12.6, 10.1; IR (film) $\nu_{\max } 2929,2859,1785,1734,1728,1233$, 1093, $1016 \mathrm{~cm}^{-1}$; HRMS (APGC) calcd. for $\mathrm{C}_{25} \mathrm{H}_{37} \mathrm{O}_{7}[\mathrm{M}+\mathrm{H}]^{+}$ 449.2539; found 449.2575.

(3S,3aR,4S)-3-Acetyloxy-7-((S)-1-((tert-butyldimethylsilyl)oxy)-2-hydroxypropan-2-yl)-1,4-dimethyl-2-oxo-2,3,3a,4,5,6hexahydroazulen-4-yl Acetate (30). Yellow oil (118 mg, 98\%); $[\alpha]_{\mathrm{D}}^{20}-79.09\left(c \mathrm{1.77}, \mathrm{CHCl}_{3}\right) ;{ }^{1} \mathrm{H}$ NMR $\left(500 \mathrm{MHz}, \mathrm{CDCl}_{3}\right) \delta 6.80$ (br s, $1 \mathrm{H}), 5.30(\mathrm{~d}, J=2.9 \mathrm{~Hz}, 1 \mathrm{H}), 4.17($ br s, $1 \mathrm{H}), 3.67(\mathrm{~d}, J=9.7$ $\mathrm{Hz}, 1 \mathrm{H}), 3.50(\mathrm{~d}, J=9.7 \mathrm{~Hz}, 1 \mathrm{H}), 2.89(\mathrm{~m}, 1 \mathrm{H}), 2.55$ (ddd, $J=14.7$, 6.4, $3.4 \mathrm{~Hz}, 1 \mathrm{H}$ ), 2.23 (ddd, $J=16.4,6.5,3.2 \mathrm{~Hz}, 1 \mathrm{H}$ ), 2.14 (ddd, $J=$ 14.1, 2.9, $2.7 \mathrm{~Hz}, 1 \mathrm{H}), 2.10(\mathrm{~s}, 3 \mathrm{H}), 1.97(\mathrm{~s}, 3 \mathrm{H}), 1.81(\mathrm{~d}, J=2.1 \mathrm{~Hz}$, $3 \mathrm{H}), 1.29(\mathrm{~s}, 3 \mathrm{H}), 1.24(\mathrm{~s}, 3 \mathrm{H}), 0.87(\mathrm{~s}, 9 \mathrm{H}), 0.07(\mathrm{~s}, 3 \mathrm{H}), 0.05(\mathrm{~s}$, $3 \mathrm{H}) ;{ }^{13} \mathrm{C}$ NMR $\left(126 \mathrm{MHz}, \mathrm{CDCl}_{3}\right) \delta 202.3,170.8,169.8,161.1$, 157.1, 135.7, 119.2, 83.0, 76.4, 73.3, 68.5, 51.8, 40.2, 25.9, 25.1, 23.9, 22.8, 22.4, 20.9, 18.3, 8.5, -5.3; IR (film) $\nu_{\max } 3447,2930,1749,1707$, 1626, 1246, 1093, $837 \mathrm{~cm}^{-1}$; HRMS (APGC) calcd. for $\mathrm{C}_{25} \mathrm{H}_{41} \mathrm{O}_{7} \mathrm{Si}$ $[\mathrm{M}+\mathrm{H}]^{+}$481.2622; found 481.2610.

(3S,3aR,4S)-3-Butanoyloxy-7-((S)-1-((tert-butyldimethylsilyl)oxy)-2-hydroxypropan-2-yl)-1,4-dimethyl-2-oxo-2,3,3a,4,5,6hexahydroazulen-4-yl Acetate (31). Yellow oil (71 mg, 56\%); $[\alpha]_{\mathrm{D}}^{20}-75.79$ (c 2.00, $\left.\mathrm{CHCl}_{3}\right)$; ${ }^{1} \mathrm{H}$ NMR $\left(400 \mathrm{MHz}, \mathrm{CDCl}_{3}\right) \delta 6.80$ (br s, $1 \mathrm{H}), 5.30$ (d, $J=2.8 \mathrm{~Hz}, 1 \mathrm{H}), 4.17$ (br s, $1 \mathrm{H}), 3.67$ (d, $J=9.7$ $\mathrm{Hz}, 1 \mathrm{H}), 3.50(\mathrm{~d}, J=9.7 \mathrm{~Hz}, 1 \mathrm{H}), 2.90(\mathrm{~m}, 1 \mathrm{H}), 2.54(\mathrm{ddd}, J=14.6$, 6.4, $3.4 \mathrm{~Hz}, 1 \mathrm{H}), 2.32(\mathrm{~m}, 2 \mathrm{H}), 2.22$ (ddd, $J=16.8,6.4,3.2 \mathrm{~Hz}, 1 \mathrm{H}$ ), 2.11 (ddd, $J=14.7,11.7,3.1 \mathrm{~Hz}, 1 \mathrm{H}), 1.96(\mathrm{~s}, 3 \mathrm{H}), 1.81(\mathrm{~d}, J=2.6$ $\mathrm{Hz}, 3 \mathrm{H}), 1.67(\mathrm{tq}, J=7.4,7.4 \mathrm{~Hz}, 2 \mathrm{H}), 1.28(\mathrm{~s}, 3 \mathrm{H}), 1.24(\mathrm{~s}, 3 \mathrm{H}), 0.97$ $(\mathrm{t}, J=7.4 \mathrm{~Hz}, 3 \mathrm{H}), 0.87(\mathrm{~s}, 9 \mathrm{H}), 0.07(\mathrm{~s}, 3 \mathrm{H}), 0.05(\mathrm{~s}, 3 \mathrm{H}) ;{ }^{13} \mathrm{C}$ NMR $\left(100 \mathrm{MHz}, \mathrm{CDCl}_{3}\right) \delta 202.4,172.5,170.8,161.1,157.1,135.7,119.2$, 83.0, 76.4, 73.1, 68.5, 51.8, 40.2, 36.0, 25.9, 25.1, 24.0, 22.8, 22.4, 18.5, 18.3, 13.8, 8.5, -5.3; IR (film) $\nu_{\max } 3496,2960,2932,2858,1743$, $1705,1626,1249,1092,836 \mathrm{~cm}^{-1}$; HRMS (APGC) calcd. for $\mathrm{C}_{27} \mathrm{H}_{44} \mathrm{O}_{7} \mathrm{Si}[\mathrm{M}]^{+}$508.2856; found 508.2859.

(3S,3aR,4S)-3-Hexanoyloxy-7-((S)-1-((tert-butyldimethylsilyl)oxy)-2-hydroxypropan-2-yl)-1,4-dimethyl-2-oxo2,3,3a,4,5,6-hexahydroazulen-4-yl Acetate (32). Yellow oil (101 $\mathrm{mg}, 75 \%) ;[\alpha]_{\mathrm{D}}^{20}-88.73\left(\mathrm{c} 2.00, \mathrm{CHCl}_{3}\right) ;{ }^{1} \mathrm{H}$ NMR $(400 \mathrm{MHz}$, $\left.\mathrm{CDCl}_{3}\right) \delta 6.79(\mathrm{br} \mathrm{s}, 1 \mathrm{H}), 5.28(\mathrm{~d}, J=2.8 \mathrm{~Hz}, 1 \mathrm{H}), 4.16(\mathrm{br} \mathrm{s}, 1 \mathrm{H})$, $3.66(\mathrm{~d}, J=9.7 \mathrm{~Hz}, 1 \mathrm{H}), 3.49(\mathrm{~d}, J=9.7 \mathrm{~Hz}, 1 \mathrm{H}), 2.89(\mathrm{~m}, 1 \mathrm{H}), 2.54$ (ddd, $J=14.5,6.2,3.2 \mathrm{~Hz}, 1 \mathrm{H}), 2.32(\mathrm{~m}, 2 \mathrm{H}), 2.22$ (ddd, $J=16.3,6.4$, $3.0 \mathrm{~Hz}, 1 \mathrm{H}), 2.11(\mathrm{ddd}, J=16.5,11.6,3.1 \mathrm{~Hz}, 1 \mathrm{H}), 1.95(\mathrm{~s}, 3 \mathrm{H}), 1.81$ $(\mathrm{d}, J=2.3 \mathrm{~Hz}, 3 \mathrm{H}), 1.63(\mathrm{~m}, 2 \mathrm{H}), 1.32(\mathrm{~m}, 4 \mathrm{H}), 1.28(\mathrm{~s}, 3 \mathrm{H}), 1.23(\mathrm{~s}$, $3 \mathrm{H}), 0.87(\mathrm{~m}, 12 \mathrm{H}), 0.06(\mathrm{~s}, 3 \mathrm{H}), 0.05(\mathrm{~s}, 3 \mathrm{H}) ;{ }^{13} \mathrm{C}$ NMR $(100 \mathrm{MHz}$, $\left.\mathrm{CDCl}_{3}\right) \delta 202.4,172.7,170.8,161.1,157.1,135.7,119.2,83.0,76.4$, 73.1, 68.5, 51.8, 40.2, 34.1, 31.3, 25.9, 25.1, 24.6, 23.9, 22.8, 22.4, 22.4, $18.3,14.0,8.5,-5.3$; IR (film) $\nu_{\max } 3488,2956,2932,2860,1743$, $1707,1625,1246,1092,838 \mathrm{~cm}^{-1}$; HRMS (APGC) calcd. for $\mathrm{C}_{29} \mathrm{H}_{49} \mathrm{O}_{7} \mathrm{Si}[\mathrm{M}+\mathrm{H}]^{+}$537.3248; found 537.3253 .

(3S,3aR,4S)-7-((S)-1-((tert-Butyldimethylsilyl)oxy)-2-hydroxypropan-2-yl)-1,4-dimethyl-3-octanoyloxy-2-oxo-2,3,3a,4,5,6hexahydroazulen-4-yl Acetate (33). Yellow oil (128 mg, 91\%);
$[\alpha]_{\mathrm{D}}^{20}-91.91$ ( c 1.53, $\left.\mathrm{CHCl}_{3}\right) ;{ }^{1} \mathrm{H}$ NMR (500 MHz, $\left.\mathrm{CDCl}_{3}\right) \delta 6.80$ $($ br s, $1 \mathrm{H}), 5.30(\mathrm{~d}, J=2.8 \mathrm{~Hz}, 1 \mathrm{H}), 4.16(\mathrm{br} \mathrm{s}, 1 \mathrm{H}), 3.67(\mathrm{~d}, J=9.7$ $\mathrm{Hz}, 1 \mathrm{H}), 3.50(\mathrm{~d}, J=9.7 \mathrm{~Hz}, 1 \mathrm{H}), 2.90(\mathrm{~m}, 1 \mathrm{H}), 2.54(\mathrm{ddd}, J=14.7$, 6.5, $3.4 \mathrm{~Hz}, 1 \mathrm{H}), 2.34(\mathrm{~m}, 2 \mathrm{H}), 2.23(\mathrm{ddd}, J=16.3,6.5,3.2 \mathrm{~Hz}, 1 \mathrm{H})$, 2.12 (ddd, $J=14.7,11.6,3.1 \mathrm{~Hz}, 1 \mathrm{H}), 1.96(\mathrm{~s}, 3 \mathrm{H}), 1.82(\mathrm{~d}, J=2.1$ $\mathrm{Hz}, 3 \mathrm{H}), 1.63(\mathrm{~m}, 2 \mathrm{H}), 1.32(\mathrm{~m}, 4 \mathrm{H}), 1.29(\mathrm{~s}, 3 \mathrm{H}), 1.27(\mathrm{~m}, 4 \mathrm{H}), 1.24$ $(\mathrm{s}, 3 \mathrm{H}), 0.88(\mathrm{~m}, 12 \mathrm{H}), 0.07(\mathrm{~s}, 3 \mathrm{H}), 0.06(\mathrm{~s}, 3 \mathrm{H}) ;{ }^{13} \mathrm{C}$ NMR $(126$ $\left.\mathrm{MHz}, \mathrm{CDCl}_{3}\right) \delta 202.4,172.7,170.8,161.1,157.1,135.8,119.2,83.0$, 76.4, 73.1, 68.5, 51.8, 40.2, 34.2, 31.8, 29.2, 29.1, 25.9, 25.1, 25.0, 24.0, 22.8, 22.7, 22.5, 18.3, 14.2, 8.5, -5.3; IR (film) $\nu_{\max } 3467,2930,2859$, $1740,1706,1624,1248,1092,836 \mathrm{~cm}^{-1}$; HRMS (APGC) calcd. for $\mathrm{C}_{31} \mathrm{H}_{53} \mathrm{O}_{7} \mathrm{Si}[\mathrm{M}+\mathrm{H}]^{+}$565.3561; found 565.3549.

For the preparation of 36 , a larger scale experiment was run for the synthesis of 33: To a suspension of $\mathrm{KMnO}_{4}(746 \mathrm{mg}, 4.720 \mathrm{mmol}$, 2.10 equiv) in dry benzene $(50 \mathrm{~mL})$ at $85^{\circ} \mathrm{C}$ were added octanoic acid (78.68 mmol, $12.5 \mathrm{~mL}, 35.0$ equiv) and its anhydride $(21.36 \mathrm{mmol}, 6.3$ $\mathrm{mL}, 9.50$ equiv). The mixture was vigorously stirred until the suspension changed from purple to brown (30 $\mathrm{min}$ ), and then, 25 (950 mg, $2.248 \mathrm{mmol}$ ) was added (dissolved in the minimum amount of dry benzene). The reaction was heated overnight at $85^{\circ} \mathrm{C}$. It is very important to keep a vigorous stirring of the reaction. Then, the mixture was cooled to room temperature and filtered through an $\mathrm{Al}_{2} \mathrm{O}_{3} /$ Celite pad rinsing with EtOAc. The solvent was evaporated under reduced pressure, and the crude oil was purified by flash column chromatography (gradient eluent from 0 to $20 \%$ EtOAc/hexanes) to lead to compound $33(1.09 \mathrm{~g}, 86 \%$ yield).

( $3 S, 3 a S, 6 R, 6 a R, 9 b S)-3,6,9$-Trimethyl-2,8-dioxo2,3,3a,4,5,6,6a,7,8,9b-decahydroazuleno[4,5-b]furan-6-yl Acetate (21). A solution of $\alpha$-santonin $20(500 \mathrm{mg}, 2.03 \mathrm{mmol})$ in acetic acid $(200 \mathrm{~mL})$ was irradiated in a quartz Hanovia reactor equipped with a $\mathrm{Hg}$ medium pressure lamp for $5 \mathrm{~h}$. Removal of the solvent under vacuum and purification of the crude mixture by flash column chromatography (40\% EtOAc/hexanes) afforded $O$-acetylisophotosantonic lactone $\mathbf{2 1}$ as a white solid (230 mg, 38\% yield). Spectroscopic data were in accordance with literature reported values. ${ }^{12}$

(3aS,4S)-7-((S)-1,2-Dihydroxypropan-2-yl)-1,4-dimethyl-2oxo-2,3,3a,4,5,6-hexahydroazulen-4-yl Acetate (24). A solution of compound $23^{13}$ (570 mg, $2.30 \mathrm{mmol}$ ) in acetic acid $(200 \mathrm{~mL})$ was irradiated and stirred in a quartz Hanovia reactor equipped with a $\mathrm{Hg}$ medium pressure lamp for $2 \mathrm{~h}$. Removal of the solvent under vacuum and purification of the crude mixture by flash column chromatography (75\% EtOAc/hexanes) afforded the product 24 as a colorless oil (510 $\mathrm{mg}, 72 \%$ yield). Spectroscopic data were in accordance with literature reported values. ${ }^{13}$

(3aS,4S)-7-((S)-1-((tert-Butyldimethylsilyl)oxy)-2-hydroxypropan-2-yl)-1,4-dimethyl-2-oxo-2,3,3a,4,5,6-hexahydroazulen-4-yl Acetate (25). Compound $24(500 \mathrm{mg}, 1.62 \mathrm{mmol})$ was dissolved in dry THF $(11 \mathrm{~mL})$, and imidazole $(330 \mathrm{mg}, 4.86 \mathrm{mmol}$, 3.00 equiv) was added. The reaction mixture was cooled to $0{ }^{\circ} \mathrm{C}$, and a solution of TBDMSCl ( $366 \mathrm{mg}, 2.43 \mathrm{mmol}, 1.50$ equiv) in dry THF $(5 \mathrm{~mL})$ was added dropwise. The reaction was stirred for $3 \mathrm{~h}$ until completion according to TLC analysis. The reaction was treated with saturated aqueous $\mathrm{NH}_{4} \mathrm{Cl}(30 \mathrm{~mL})$ and extracted with $\mathrm{Et}_{2} \mathrm{O}(3 \times 15$ $\mathrm{mL}$ ). The combined organic layers were washed with brine and dried over anhydrous $\mathrm{Na}_{2} \mathrm{SO}_{4}$, and the solvent was evaporated under reduced pressure. Purification by flash column chromatography $(30 \%$ EtOAc/hexanes) afforded 25 as a yellow oil $(678 \mathrm{mg}, 99 \%$ yield $)$ : $[\alpha]_{\mathrm{D}}^{20}+28.19\left(\mathrm{c} 1.73, \mathrm{CHCl}_{3}\right) ;{ }^{1} \mathrm{H} \mathrm{NMR}\left(500 \mathrm{MHz}, \mathrm{CDCl}_{3}\right) \delta 6.77(\mathrm{~s}$, $1 \mathrm{H}), 4.14(\mathrm{br} \mathrm{s}, 1 \mathrm{H}), 3.68(\mathrm{~d}, J=9.6 \mathrm{~Hz}, 1 \mathrm{H}), 3.49(\mathrm{~d}, J=9.7 \mathrm{~Hz}$, $1 \mathrm{H}), 2.93(\mathrm{~m}, 1 \mathrm{H}), 2.59$ (ddd, $J=15.1,6.9,2.7 \mathrm{~Hz}, 1 \mathrm{H}), 2.42(\mathrm{dd}, J=$ $19.1,2.6 \mathrm{~Hz}, 1 \mathrm{H}$ ), 2.37 (dd, $J=19.2,2.6 \mathrm{~Hz}, 1 \mathrm{H}), 2.23$ (ddd, $J=17.1$, 6.8, $2.5 \mathrm{~Hz}, 1 \mathrm{H}), 2.00(\mathrm{~s}, 3 \mathrm{H}), 1.94(\mathrm{ddd}, J=14.8,11.9,2.7 \mathrm{~Hz}, 1 \mathrm{H})$, $1.78(\mathrm{~d}, J=1.8 \mathrm{~Hz}, 3 \mathrm{H}), 1.28(\mathrm{~s}, 3 \mathrm{H}), 1.13(\mathrm{~s}, 3 \mathrm{H}), 0.87(\mathrm{~s}, 9 \mathrm{H}), 0.07$ $(\mathrm{s}, 3 \mathrm{H}), 0.06(\mathrm{~s}, 3 \mathrm{H}) ;{ }^{13} \mathrm{C} \mathrm{NMR}\left(126 \mathrm{MHz}, \mathrm{CDCl}_{3}\right) \delta 208.5,170.7$, 163.5 , 156.9, 137.7, 119.6, 84.7, 76.3, 68.5, 45.9, 40.1, 37.3, 25.9, 25.1, 22.9, 22.6, 22.4, 18.3, 8.4, -5.3; IR (film) $\nu_{\max } 3457,2930,2857,1732$, 1694, 1627, 1464, 1368, 1250, 1093, $837 \mathrm{~cm}^{-1}$; HRMS (APGC) calcd. for $\mathrm{C}_{23} \mathrm{H}_{39} \mathrm{O}_{5} \mathrm{Si}[\mathrm{M}+\mathrm{H}]^{+}$423.2567; found 423.2587 .

(1S,8S,8aR)-8-Acetoxy-5-((S)-1,2-dihydroxypropan-2-yl)-3,8dimethyl-2-oxo-1,2,6,7,8,8a-hexahydroazulen-1-yl Octanoate 
(34). To a solution of compound $33(163 \mathrm{mg}, 0.288 \mathrm{mmol})$ in dry THF $(2 \mathrm{~mL})$ at $0{ }^{\circ} \mathrm{C}$ was added TBAF $(1 \mathrm{M}$ solution in THF, 0.575 $\mathrm{mL}, 0.575 \mathrm{mmol}, 2.00$ equiv) dropwise. The mixture was stirred at room temperature until completion according to TLC analysis $(2 \mathrm{~h})$. The reaction was treated with saturated aqueous $\mathrm{NH}_{4} \mathrm{Cl}(5 \mathrm{~mL})$ and extracted with EtOAc $(3 \times 5 \mathrm{~mL})$. Combined organic layers were washed with brine and dried over anhydrous $\mathrm{Na}_{2} \mathrm{SO}_{4}$, and the solvent was removed under vacuum. Purification by flash column chromatography (70\% EtOAc/hexanes) afforded product 34 as a yellow oil (74 $\mathrm{mg}, 61 \%$ yield): $[\alpha]_{\mathrm{D}}^{20}-92.32\left(c 1.92, \mathrm{CHCl}_{3}\right) ;{ }^{1} \mathrm{H}$ NMR (500 MHz, $\left.\mathrm{CDCl}_{3}\right) \delta 6.87(\mathrm{~s}, 1 \mathrm{H}), 5.25(\mathrm{~d}, J=2.8 \mathrm{~Hz}, 1 \mathrm{H}), 4.22(\mathrm{br} \mathrm{s}, 1 \mathrm{H}), 3.75$ $(\mathrm{d}, J=11.0 \mathrm{~Hz}, 1 \mathrm{H}), 3.52(\mathrm{~d}, J=11.0 \mathrm{~Hz}, 1 \mathrm{H}), 2.94(\mathrm{~m}, 1 \mathrm{H}), 2.80(\mathrm{br}$ s, $O H), 2.57(\mathrm{ddd}, J=14.8,6.4,3.4 \mathrm{~Hz}, 1 \mathrm{H}), 2.33(\mathrm{~m}, 2 \mathrm{H}), 2.21$ (ddd, $J=16.4,6.4,3.2 \mathrm{~Hz}, 1 \mathrm{H}), 2.11$ (ddd $J=14.8,11.7,3.1 \mathrm{~Hz}, 1 \mathrm{H}), 1.96$ $(\mathrm{s}, 3 \mathrm{H}), 1.82(\mathrm{~d}, J=2.1 \mathrm{~Hz}, 3 \mathrm{H}), 1.62(\mathrm{tt}, J=7.4,7.4 \mathrm{~Hz}, 2 \mathrm{H}), 1.34(\mathrm{~s}$, $3 \mathrm{H}), 1.28(\mathrm{~m}, 8 \mathrm{H}), 1.24(\mathrm{~s}, 3 \mathrm{H}), 0.86(\mathrm{t}, J=7.0 \mathrm{~Hz}, 3 \mathrm{H}) ;{ }^{13} \mathrm{C} \mathrm{NMR}$ $\left(126 \mathrm{MHz} \mathrm{CDCl}_{3}\right) \delta 202.6,172.8,170.8,160.9,156.8,136.0,119.6$, 83.0, 77.4, 73.3, 68.1, 51.7, 40.3, 34.1, 31.8, 29.2, 29.1, 25.2, 24.9, 24.1, 23.0, 22.7, 22.5, 14.2, 8.6; IR (film) $\nu_{\max } 3449,2931,2858,1739,1704$, $1623,1370,1244,1163,1045 \mathrm{~cm}^{-1}$; HRMS (ESI) calcd. for $\mathrm{C}_{25} \mathrm{H}_{37} \mathrm{O}_{7}$ $[\mathrm{M}-\mathrm{H}]^{-}$449.2539; found 449.2539 .

(3S,3aR,6S,6aR,7S,9bS)-6-Acetoxy-3,3a-dihydroxy-3,6,9-trimethyl-2,8-dioxo-2,3,3a,4,5,6,6a,7,8,9b-decahydroazuleno[4,5-b]furan-7-yl Octanoate (36). Guaianediol $34(70 \mathrm{mg}, 0.155$ $\mathrm{mmol})$ was dissolved in a $20: 2.5: 1$ acetone $/ \mathrm{tBuOH} / \mathrm{H}_{2} \mathrm{O}$ solution $(8$ $\mathrm{mL}$, conc. $0.02 \mathrm{M})$ and then cooled at $0{ }^{\circ} \mathrm{C} . \mathrm{MeSO}_{2} \mathrm{NH}_{2}(18 \mathrm{mg}$, $0.186 \mathrm{mmol}, 1.20$ equiv), NMO (22 $\mathrm{mg}, 0,186 \mathrm{mmol}, 1.20$ equiv), and $\mathrm{OsO}_{4}(2 \mathrm{mg}, 0.008 \mathrm{mmol}, 0.05$ equiv) were added. The reaction was stirred for $24 \mathrm{~h}$ at room temperature and then treated with saturated aqueous $\mathrm{Na}_{2} \mathrm{SO}_{3}$ solution $(5 \mathrm{~mL})$. After stirring for $40 \mathrm{~min}$, water $(5$ $\mathrm{mL})$ was added and the mixture was extracted with EtOAc $(4 \times 10$ $\mathrm{mL}$ ). The combined organic layers were dried over anhydrous $\mathrm{Na}_{2} \mathrm{SO}_{4}$, and the solvent was removed under reduced pressure. This material $(75 \mathrm{mg})$ was used without further purification in the subsequent step. Crude compound 35 (ca. $75 \mathrm{mg}, 0.155 \mathrm{mmol}$ ) was dissolved in a $1.3: 1 \mathrm{MeCN} / \mathrm{pH} 7$ buffer solution mixture $(15 \mathrm{~mL})$. TEMPO ( $13 \mathrm{mg}, 0.078 \mathrm{mmol}, 0.50$ equiv), $\mathrm{NaClO}_{2}$ ( $0.224 \mathrm{~mL}, 0.620$ mmol, 4.00 equiv, $25 \% \mathrm{w} / \mathrm{w}$ in $\left.\mathrm{H}_{2} \mathrm{O}\right)$, and $\mathrm{NaClO}(0.04 \mathrm{~mL})$ were added. The mixture was stirred at room temperature for $4 \mathrm{~h}$, and then aqueous saturated $\mathrm{Na}_{2} \mathrm{SO}_{3}$ solution $(4 \mathrm{~mL})$ was added. The $\mathrm{pH}$ was set to 3 by adding aqueous $2 \mathrm{~N} \mathrm{HCl}$ solution. The mixture was extracted with EtOAc $(4 \times 10 \mathrm{~mL})$ and washed with brine $(15 \mathrm{~mL})$. After drying over anhydrous $\mathrm{Na}_{2} \mathrm{SO}_{4}$, the solvent was removed under reduced pressure, affording pure compound $\mathbf{3 6}$ as a white amorphous solid (48 mg, 65\%): $[\alpha]_{\mathrm{D}}^{20}-166.5\left(c 0.30, \mathrm{CH}_{3} \mathrm{Cl}\right) ;{ }^{1} \mathrm{H}$ NMR (400 $\left.\mathrm{MHz}, \mathrm{CDCl}_{3}\right) \delta 5.44(\mathrm{br} \mathrm{s}, 1 \mathrm{H}), 5.14(\mathrm{~d}, J=3.6 \mathrm{~Hz}, 1 \mathrm{H}), 4.34(\mathrm{br} \mathrm{s}$, $1 \mathrm{H}), 2.78(\mathrm{~m}, 1 \mathrm{H}), 2.59(\mathrm{br} \mathrm{s}, \mathrm{OH}), 2.34(\mathrm{~m}, 2 \mathrm{H}), 2.25$ (ddd, $J=13.7$, $3.9,3.9 \mathrm{~Hz}, 1 \mathrm{H}), 2.01(\mathrm{~m}, 1 \mathrm{H}), 1.97(\mathrm{~d}, J=2.5 \mathrm{~Hz}, 3 \mathrm{H}), 1.95(\mathrm{~s}, 3 \mathrm{H})$, 1.88 (ddd, $J=15.6,4.2,4.2 \mathrm{~Hz}, 1 \mathrm{H}), 1.63(\mathrm{~m}, 4 \mathrm{H}), 1.44(\mathrm{~s}, 3 \mathrm{H}), 1.31$ $(\mathrm{s}, 3 \mathrm{H}), 1.27(\mathrm{~m}, 6 \mathrm{H}), 0.86(\mathrm{t}, J=6.7 \mathrm{~Hz}, 3 \mathrm{H}) ;{ }^{13} \mathrm{C} \mathrm{NMR}(100 \mathrm{MHz}$, $\left.\mathrm{CDCl}_{3}\right) \delta 201.4,175.6,172.8,170.6,156.2,141.7,84.7,82.5,80.1$, 77.8, 73.8, 52.7, 34.0, 31.8, 31.7, 29.1, 29.0, 24.9, 24.6, 22.8, 22.7, 19.9, 15.9, 14.2, 10.2; IR (film) $\nu_{\max } 3438,2957,2928,2858,1794,1727$, $1369,1260,1095,1018,804 \mathrm{~cm}^{-1}$; HRMS (ESI) calcd. for $\mathrm{C}_{25} \mathrm{H}_{35} \mathrm{O}_{9}$ $[\mathrm{M}-\mathrm{H}]^{-}$479.2281; found 479.2287.

\section{ASSOCIATED CONTENT}

\section{S Supporting Information}

Spectroscopic data for all compounds. This material is available free of charge via the Internet at http://pubs.acs.org.

\section{AUTHOR INFORMATION}

\section{Corresponding Authors}

*E-mail: francisco.guerra@uca.es (F.M.G).

*E-mail: javi.moreno@uca.es (F.J.M.-D.).

\section{Notes}

The authors declare no competing financial interest.

\section{ACKNOWLEDGMENTS}

This research was supported by Junta de Andalucía (FQM169). The authors are grateful to Prof. Dr. M. J. Ortega for her assistance with the MS analyses and to the Servicios Centrales de Ciencia y Tecnología (SCCyT) of the University of Cádiz. R.M.-B. and A.L.G.-C. thank the Spanish Ministry of Education, Culture and Sport for fellowships.

\section{REFERENCES}

(1) White, M. C. Synlett 2012, 23, 2746-2748.

(2) (a) Shilov, A. E.; Shul'pin, G. B. Chem. Rev. 1997, 97, 28792932. (b) Crabtree, R. H. J. Chem. Soc., Dalton Trans. 2001, 24372450. (c) Davies, H. M. L.; Manning, J. R. Nature 2008, 451, 417-424. (d) Gutekunst, W. R.; Baran, P. S. Chem. Soc. Rev. 2011, 40, 19761991. (e) Newhouse, T.; Baran, P. S. Angew. Chem., Int. Ed. 2011, 50, 3362-3374. (f) Yamaguchi, J.; Yamaguchi, A. D.; Itami, K. Angew. Chem., Int. Ed. 2012, 51, 8960-9009.

(3) (a) Eames, J.; Watkinson, M. Angew. Chem., Int. Ed. 2001, 40, 3567-3571. (b) Andrus, M. B.; Lashley, J. C. Tetrahedron 2002, 58, 845-866. (c) Chen, M. S.; White, M. C. J. Am. Chem. Soc. 2004, 126, 1346-1347. (d) Delcamp, J. H.; White, M. C. J. Am. Chem. Soc. 2006, 128, 15076-15077. (e) McLaughlin, E. C.; Choi, H.; Wang, K.; Chiou, G.; Doyle, M. P. J. Org. Chem. 2009, 74, 730-738. (f) Marín-Barrios, R; Guerra, F. M.; García-Cabeza, A. L.; Moreno-Dorado, F. J.; Massanet, G. M. Tetrahedron 2012, 68, 1105-1108. (g) Weidman, V.; Maison, W. Synthesis 2013, 45, 2201-2221. (h) García-Cabeza, A. L.; Marín-Barrios, R.; Azarken, R; Moreno-Dorado, F. J.; Ortega, M. J.; Vidal, H.; Gatica, J. M.; Massanet, G. M.; Guerra, F. M. Eur. J. Org. Chem. 2013, 8307-8314. (i) García-Cabeza, A. L.; Marín-Barrios, R.; Moreno-Dorado, F. J.; Ortega, M. J.; Massanet, G. M.; Guerra, F. M. Org. Lett. 2014, 16, 1598-1601.

(4) (a) Merino, P.; Tejero, T. Angew. Chem., Int. Ed 2004, 43, 29952997. (b) Adam, W.; Lazarus, M.; Saha-Möller, C. R.; Schreier, P. Acc. Chem. Res. 1999, 32, 837-845. (c) Davis, F. A.; Chen, B.-C. In Stereoselective Synthesis; Helmchen, G., Hoffmann, R. W., Muzler, J., Schaumann, E., Eds.; Houben-Weyl Methods of Organic Chemistry; George Thieme: Stuttgart, 1996; Vol. E21. (d) Fuhrhop, J.; Penalin, G. Organic Synthesis, 2nd ed.; VCH: Weinheim, 1994. (e) Davis, F. A.; Chen, B.-C. Chem. Rev. 1992, 92, 919-934. (f) Solujic, S.; Sukdolak, S.; Ratkovic, Z. Tetrahedron Lett. 1991, 32, 4577-4578. (g) Williams, G. J.; Hunter, N. R. Can. J. Chem. 1976, 54, 3830-3832.

(5) (a) Demir, A. S.; Jeganathan, A.; Watt, D. S. J. Org. Chem. 1989, 54, 4020-4022. (b) Voyle, M.; Dunlap, N. K.; Watt, D. S. J. Org. Chem. 1983, 48, 3242-3245. (c) Dunlap, N. K.; Sabol, M. R.; Watt, D. S. Tetrahedron Lett. 1984, 25, 5839-5842.

(6) Demir, A. S.; Reis, O.; Igdir, C. Tetrahedron 2004, 60, 34273432.

(7) Heiba, E. I.; Dessau, R. M.; Koehl, W. J. J. Am. Chem. Soc. 1969, 91, 138-145.

(8) Demir, A. S.; Findik, H. Tetrahedron 2008, 64, 6196-6201.

(9) For a general introduction to experimental design in chemistry, see: (a) Araujo, P. W.; Brereton, R. G. TrAC, Trends Anal. Chem. 1996, 15, 26-31. (b) Araujo, P. W.; Brereton, R. G. TrAC, Trends Anal. Chem. 1996, 15, 63-70. (c) Carlson, R.; Carlson, J. E. Design and Optimization in Organic Synthesis. In Data Handling and in Science and Technology; Elsevier: Amsterdam, 2005. (d) Bezerra, M. A.; Santelli, R. E.; Oliveira, E. P.; Villar, L. S.; Escaleira, L. A. Talanta 2008, 76, 965-977. (e) Leardy, R. Anal. Chim. Acta 2009, 652, 161172. (f) Murray, P. M.; Tyler, S. N. G.; Moseley, J. D. Org. Process Res. Dev. 2013, 17, 40-46.

(10) (a) Lytton, J.; Westlin, M.; Hanley, M. R. J. Biol. Chem. 1991, 266, 17067-17071. (b) Inesi, G.; Sagara, Y. Arch. Biochem. Biophys. 1992, 298, 313-317. (c) Christensen, S. B.; Andersen, A.; Smitt, U. W. Prog. Chem. Org. Nat. Prod. 1997, 71, 130-167. (d) Treiman, M.; Carpersen, C.; Christensen, S. B. Trends Pharmacol. Sci. 1998, 19, 131-135. (e) Christensen, S. B.; Andersen, A.; Kromann, H.; Treiman, M.; Tombal, B.; Denmeade, S. R.; Isaacs, J. T. Biorg. Med. Chem. 1999, 7, 1273-1280. (f) Jacobsen, C. M.; Denmeade, S. R.; 
Isaacs, J. T.; Gady, A.; Olsen, C. E.; Christensen, S. B. J. Med. Chem. 2001, 44, 4696-4703.

(11) Baghbanzadeh, M.; Kappe, C. O. Aust. J. Chem. 2009, 62, 244249.

(12) Zang, W.; Luo, S.; Fang; Chen, Q.; Hu, H.; Jia, X.; Zhai, H. J. Am. Chem. Soc. 2005, 127, 18-19.

(13) Manzano, F. L.; Guerra, F. M.; Moreno-Dorado, F. J.; Jorge, D. Z.; Massanet, G. M. Org. Lett. 2006, 8, 2879-2882.

(14) Ghantous, A.; Gali-Muhtasib, H.; Vuorela, H.; Saliba, N. A.; Darwiche, N. Drug Discovery Today 2010, 15, 668-678.

(15) (a) Oliver, S. F.; Högenauer, K.; Simic, O.; Antonello, A.; Smith, M. D.; Ley, S. V. Angew. Chem., Int. Ed. 2003, 42, 5996-6000. (b) Ley, S. V.; Antonello, A.; Balskus, E. P.; Booth, D. T.; Christensen, S. B.; Cleator, E.; Gold, H.; Högenauer, K.; Hünger, U.; Myers, R. M.; Oliver, S. F.; Simic, O.; Smith, M. D.; Søhoel, H.; Wooldford, A. J. A. Proc. Natl. Acad. Sci. U.S.A. 2004, 101, 12073-12078. (c) Ball, M.; Andrews, S. P.; Wierschem, F.; Cleator, E.; Smith, M. D.; Ley, S. V. Org. Lett. 2007, 9, 663-666. (d) Andrews, S. P.; Ball, M.; Wierschem, F.; Cleator, E.; Oliver, S.; Högenauer, K.; Simic, O.; Antonello, A.; Hünger, U.; Smith, M. D.; Ley, S. V. Chem.-Eur. J. 2007, 13, 56885712.

(16) (a) Søhoel, H.; Liljefors, T.; Ley, S. V.; Oliver, S. F.; Antonello, A.; Smith, M. D.; Olsen, C. E.; Isaacs, J. T.; Christensen, S. B. J. Med. Chem. 2005, 48, 7005-7011. (b) Andrews, S. P.; Tait, M. M.; Ball, M.; Ley, S. V. Org. Biomol. Chem. 2007, 5, 1427-1436.

(17) (a) Denmeade, S. R.; Jacobsen, C. M.; Janssen, S.; Khan, S. R.; Garrett, E. S.; Lilja, H.; Christensen, S. B.; Isaacs, J. T. J. Natl. Cancer Inst. 2003, 13, 990-1000. (b) Isaacs, J. T. BJU Int. 2005, 96, 35-40.

(18) Denmeade, R. S.; Mhaka, A. M.; Rosen, D. M.; Brennen, W. N.; Dalrymple, S.; Dach, I.; Olesen, C.; Gurel, B.; DeMarzo, A. M.; Wilding, G.; Carducci, M. A.; Dionne, C. A.; Møller, J. V.; Nissen, P.; Christensen, S. B.; Isaacs, J. T. Sci. Transl. Med. 2012, 4, 14086. 